\title{
Low dose radiation risks for women surviving the a-bombs in Japan: generalized additive model
}

\author{
Greg Dropkin (1)
}

\begin{abstract}
Background: Analyses of cancer mortality and incidence in Japanese A-bomb survivors have been used to estimate radiation risks, which are generally higher for women. Relative Risk (RR) is usually modelled as a linear function of dose. Extrapolation from data including high doses predicts small risks at low doses. Generalized Additive Models (GAMs) are flexible methods for modelling non-linear behaviour.

Methods: GAMs are applied to cancer incidence in female low dose subcohorts, using anonymous public data for the 1958 - 1998 Life Span Study, to test for linearity, explore interactions, adjust for the skewed dose distribution, examine significance below $100 \mathrm{mGy}$, and estimate risks at $10 \mathrm{mGy}$.

Results: For all solid cancer incidence, RR estimated from 0 - $100 \mathrm{mGy}$ and 0 - $20 \mathrm{mGy}$ subcohorts is significantly raised. The response tapers above $150 \mathrm{mGy}$. At low doses, RR increases with age-at-exposure and decreases with time-since-exposure, the preferred covariate. Using the empirical cumulative distribution of dose improves model fit, and capacity to detect non-linear responses. RR is elevated over wide ranges of covariate values. Results are stable under simulation, or when removing exceptional data cells, or adjusting neutron RBE. Estimates of Excess RR at 10 mGy using the cumulative dose distribution are $10-45$ times higher than extrapolations from a linear model fitted to the full cohort. Below 100 mGy, quasipoisson models find significant effects for all solid, squamous, uterus, corpus, and thyroid cancers, and for respiratory cancers when age-at-exposure $>35 \mathrm{yrs}$. Results for the thyroid are compatible with studies of children treated for tinea capitis, and Chernobyl survivors. Results for the uterus are compatible with studies of UK nuclear workers and the Techa River cohort.
\end{abstract}

Conclusion: Non-linear models find large, significant cancer risks for Japanese women exposed to low dose radiation from the atomic bombings. The risks should be reflected in protection standards.

Keywords: Low dose radiation, Cancer, Women, Thyroid, Uterus, A-bomb, GAM

\section{Background}

Analyses of cancer mortality and incidence in Japanese Abomb survivors continue to play a key role in establishing radiation risk estimates. The 1958-1998 incidence cohort was analysed by Preston and co-workers [1], whose main model assumes a linear dose response. Their study found some evidence of radiation risk at doses below $150 \mathrm{mGy}$, and higher risks for women. Previously Pierce and Preston [2] found a significant dose response for all solid cancer incidence in the $0-100$ mGy range. However,

Correspondence: gregd@gn.apc.org

Independent researcher, Liverpool, England according to the EPA Blue Book [3] "it has not been possible to demonstrate and quantify risk in the Lifespan Study (of A-bomb survivors) at doses below about 100 mGy". The ICRP [4] recommended annual dose limit for occupational exposures is $20 \mathrm{mSv}$ (whole body dose).

Some cancer studies find non-linear responses to radiation [5-8] and there are some significant results at low doses [9-16]. Low dose radiation risk was reviewed in 2003 [17] and remains a focus of research [18].

I consider cancer incidence in female Japanese A-bomb survivors in the dose ranges $0-500 \mathrm{mGy}, 0-100 \mathrm{mGy}$, and $0-20 \mathrm{mGy}$, using the grouped anonymous (public) incidence data for the 1958 - 1998 cohort. 
Attained age is the single most powerful predictor of cancer incidence. Models can be refined using birth cohort, age-at-exposure or time-since-exposure, city, urban/rural residence, and dose. The Not In City (NIC) group of Hiroshima and Nagasaki residents who were out of the area at the time of bombing can be included.

The analysis uses the Generalized Additive Model [19] implemented in $\mathrm{R}$ [20] through the programme mgcv [21]. GAM is a flexible technique for fitting non-linear responses and covariate interactions. Models are investigated for all solid cancers, and then applied to the most common anatomical or histological groups and specific sites within the female incidence data: digestive, genital, respiratory, adenocarcinoma, other epithelial, squamous, stomach, colon, liver, pancreas, gallbladder, rectum, uterine, cervical, uterine corpus, ovarian, lung, breast, thyroid, and central nervous system.

The analysis aims to test for linearity of the dose response, explore the interaction of dose with attained age, age-at-exposure and time-since-exposure, adjust for the highly skewed distribution of dose, test whether the response is significant below $100 \mathrm{mGy}$, and estimate the risks at $10 \mathrm{mGy}$.

\section{Methods}

The public dataset LSSinc07 [22] was downloaded from the Radiation Effects Research Foundation (RERF) [23]. Records with unknown dose were excluded, leaving 25,570 cells with grouped data on 17,448 solid cancers among 105,427 survivors with 2,764,735 person-years of follow-up. The covariate py denotes person-years observation in each cell. logage is the log of age, the attainedage covariate which RERF obtained by weighting each individual contribution by its p-y within the cell. Likewise agex is the $p-y$ weighted mean age-at-exposure, since is the difference between year, the $\mathrm{p}-\mathrm{y}$ weighted mean calendar time, and the exposure date (depending on city), and birth is defined as year - age. The dataset also contains sex, city, and distcat, the latter taking the values 1 (urban), 2 (rural), and 3 (Not in City). The grouped data is stratified by these (and other) categories. The dataset is then restricted by sex $=2$, and by distcat $<3$ if the NIC group is excluded; city and distcat are converted to factor variables fc and fd. For convenience, covariate names are introduced in bold, but then used in normal font.

When modelling all solid cancers, the instantaneous flash dose is the p-y weighted mean colon dose in $\mathrm{mGy}$, defined as coldose $=1000 *$ cola02w10 using the DS02 dosimetry and a Relative Biological Effectiveness (for neutrons) of 10, unless otherwise specified. The dataset is then restricted by coldose $<100$ (or $<20$ or $<$ $500)$. For convenience $\mathrm{A}^{+-}, \mathrm{B}^{+-}$and $\mathrm{C}^{+-}$refer to $0-$
20 mGy, 0 - 100 mGy and 0 - 500 mGy subcohorts including or excluding NIC. Weighting each coldose by the py of its data cell, the mean coldose in $\mathrm{A}^{+-}, \mathrm{B}^{+-}$ and $\mathrm{C}^{+-}$is $2.43,3.70,9.84,13.85,36.44,48.93 \mathrm{mGy}$ respectively.

More generally, modelling uses the appropriate organ dose as in [1] and LSSinc07. Tissue weighting factors are not applied. When considering a cancer group or site, dose refers to the relevant $\mathrm{p}-\mathrm{y}$ weighted organ dose in mGy, and the response variable obs is the number of incident cases of that cancer in each data cell. The case numbers by site and dose range are shown in Table 1 . Note that $\mathrm{A}^{+-}$etc. are defined from coldose, no matter which cancer site is under consideration.

Modelling seeks to predict the cancer risk, i.e. the expected value of obs/py, as a function of covariates and their interactions. Each data cell is regarded as an independent source of Poisson distributed incident cases, and penalised regression with cubic splines, using a log link, is used to estimate the expected value of obs. Briefly (see [21]):

obs $_{\mathbf{j}} \sim \operatorname{Pois}\left(\mu_{\mathrm{j}}\right)$ where $\mathrm{j}$ indexes the data cells

$\log \left(\mu_{\mathrm{j}}\right)=\sum_{\mathrm{i}} \beta_{\mathrm{i}} \mathrm{f}_{\mathrm{i}}\left(\mathrm{x}_{\mathrm{j}}\right)$ where $\mathrm{i}$ indexes the model components $\mathrm{f}$ and coefficients $\beta$; $\mathrm{f}_{\mathrm{i}}$ is a function of covariates $\mathrm{x}$, whose form is specified in the model but whose value varies with $\mathrm{x}_{\mathrm{j}}$ in cell $\mathrm{j}$; and $\beta_{\mathrm{i}}$ is a numerical coefficient of $f_{i}$, independent of $j$, to be estimated during model fitting.

The $\mathrm{f}_{\mathrm{i}}$ can include offsets, whose $\beta_{\mathrm{i}}$ is fixed at 1 ; factors; parametric terms; splines derived from the covariates; and tensor products of such splines.

A cubic regression spline with components $f_{0}, f_{1} \ldots f_{k-1}$ is obtained from covariate $\mathrm{x}$ by placing $\mathrm{k}$ knots, for example at equally spaced quantiles of $\mathrm{x}$, forming the natural cubic splines with value 1 at the specified knot and 0 at the other knots, using a centering constraint to achieve $\sum \mathrm{f}_{\mathrm{i}}\left(\mathrm{x}_{\mathrm{j}}\right)=0$ when $\mathrm{i}>0$, and setting $\mathrm{f}_{0}=1$.

During model fitting, the $\beta_{\mathrm{i}}$ are estimated by minimising the penalised deviance, Dev $+\beta^{\mathrm{T}} \mathrm{S} \beta$, where the positive semi-definite penalty matrix $S$ is a direct sum of blocks generated from the splines, each block incorporating a smoothing parameter $\lambda_{\mathrm{b}}$. For tensor product splines, there are separate smoothing parameters for each margin. Dev is the Deviance, given in the Poisson case (omitting subscripts $\mathrm{j}$ ) by

$$
\begin{aligned}
& -2 \sum\left[\mathrm{obs} \cdot \log (\mu)-\mu-\mathrm{I}_{\mathrm{obs}} \cdot(\mathrm{obs} \cdot \log (\mathrm{obs})-\mathrm{obs})\right] \\
& \text { where } \mathrm{I}_{\mathrm{obs}}=1 \text { if obs }>0 \text { and } 0 \text { otherwise }
\end{aligned}
$$

Minimising the penalised deviance is a compromise between approximating the data (lowering Dev), and the "wiggliness" of the fitted model as measured by the penalty. Higher values of the smoothing parameter(s) impose stronger penalties, making the fitted model smoother with lower "effective degrees of freedom" (a penalised version of the number of independent parame- 
Table 1 Case numbers by site and dose range

\begin{tabular}{|c|c|c|c|c|c|c|c|c|c|}
\hline Site & $A^{-}$ & $\mathrm{A}^{+}$ & $\mathrm{B}^{-}$ & $\mathrm{B}^{+}$ & $\mathrm{C}^{-}$ & $\mathrm{C}^{+}$ & Mid age & Mid agex & Mid since \\
\hline Solid & 4239 & 6271 & 5474 & 7506 & 6712 & 8744 & 68.4 & 33.1 & 36.9 \\
\hline Adeno & 2477 & 3722 & 3188 & 4433 & 3928 & 5173 & 67.0 & 28.3 & 38.6 \\
\hline Othepi & 1016 & 1460 & 1310 & 1754 & 1583 & 2027 & 73.3 & 38.4 & 32.7 \\
\hline Squam & 550 & 823 & 718 & 991 & 881 & 1154 & 63.5 & 33.2 & 28.6 \\
\hline Digest & 2206 & 3263 & 2825 & 3882 & 3418 & 4475 & 71.7 & 33.5 & 38.6 \\
\hline Genital & 660 & 1008 & 871 & 1219 & 1035 & 1383 & 61.8 & 31.6 & 28.6 \\
\hline Resp & 324 & 487 & 441 & 604 & 558 & 721 & 73.2 & 36.5 & 38.6 \\
\hline Stom & 997 & 1459 & 1285 & 1747 & 1564 & 2026 & 71.6 & 36.6 & 33.6 \\
\hline Colon & 373 & 578 & 471 & 676 & 570 & 775 & 71.7 & 29.3 & 44.1 \\
\hline Liver & 287 & 416 & 356 & 485 & 433 & 562 & 71.7 & 32.6 & 41.4 \\
\hline Gall & 181 & 266 & 234 & 319 & 273 & 358 & 73.2 & 36.8 & 38.6 \\
\hline Panc & 134 & 192 & 172 & 230 & 208 & 266 & 73.4 & 36.6 & 38.6 \\
\hline Rect & 183 & 283 & 245 & 345 & 291 & 391 & 68.5 & 31.6 & 41.3 \\
\hline Uterus & 519 & 806 & 689 & 976 & 821 & 1108 & 61.7 & 31.5 & 28.6 \\
\hline Cervix & 384 & 598 & 509 & 723 & 603 & 817 & 61.8 & 31.7 & 28.5 \\
\hline Corpus & 84 & 132 & 108 & 156 & 126 & 174 & 61.1 & 23.1 & 38.5 \\
\hline Ovary & 115 & 170 & 143 & 198 & 171 & 226 & 63.5 & 31.6 & 33.6 \\
\hline Breast & 441 & 667 & 554 & 780 & 713 & 939 & 59.1 & 22.4 & 38.6 \\
\hline Lung & 290 & 437 & 396 & 543 & 503 & 650 & 73.3 & 36.4 & 38.6 \\
\hline Thyroid & 158 & 219 & 203 & 264 & 271 & 332 & 66.7 & 28.2 & 31.9 \\
\hline Cns & 93 & 117 & 125 & 149 & 151 & 175 & 66.2 & 26.7 & 37.0 \\
\hline
\end{tabular}

Mid age (agex, since) is the age (agex, since) below which $50 \%$ of cases occur.

Values are shown for $\mathrm{B}^{+}$, and are similar for other dose ranges

ters), but allowing it to stray further from the individual data points. As $\lambda_{\mathrm{b}} \rightarrow \infty$, the model becomes log-linear (only the linear components of the spline terms are unpenalised by S).

Model fitting involves optimising the smoothing parameters affecting $\mathrm{S}$, through a criterion such as Generalized Cross Validation, Restricted Maximum Likelihood, or Marginal Likelihood (see [21, 24] for details). After fitting, inference is based on the Bayesian posterior covariance of $\beta$.

With the fitted $\hat{\beta}_{i}$, the expected value of obs is $\mu_{j}=$ $\exp \left(\sum_{\mathrm{i}} \hat{\beta}_{\mathrm{i}} \mathrm{f}_{\mathrm{i}}\left(\mathrm{x}_{\mathrm{j}}\right)\right)$. The fitted model also predicts the expected value $\mu_{\mathrm{j}, 0}$ if the dose covariate is reset to 0 while preserving all other covariates and the $\hat{\beta}_{\mathrm{i}}$. In each cell, baseline risk is estimated internally from the fitted model as $\mu_{\mathrm{j}, 0} / \mathrm{py}_{\mathrm{j}}$ and Relative Risk is $\mathrm{RR}_{\mathrm{j}}=\mu_{\mathrm{j}} / \mu_{\mathrm{j}, 0}$.

From here on, models are defined and fitted using the $\mathrm{R}$ programme mgcv. For example, the initial Poisson model is specified as

$$
\begin{aligned}
P 0< & -g a m\left(\text { obs } \sim \text { offset }(\log (p y))+(f c+f d)^{\wedge} 2\right. \\
& + \text { ti }(\text { logage }, k=10)+\text { ti }(\text { birth }, k=10) \\
& + \text { ti }(\text { logage }, \text { birth }, k=10), \\
& \text { family }=\text { "poisson", method }=\text { "ML" })
\end{aligned}
$$

The reverse arrow $<$ - defines the model by the subsequent expression, with gam denoting a generalized additive model. Here, obs is regarded as a sample from a Poisson distribution whose mean, varying across the data cells, is specified with a (default) log link. The offset term, with coefficient fixed at 1 , ensures that the fitted value of obs/py is independent of py. The factor variables and their first order interaction are included as $\left(\mathrm{fc}_{\mathrm{c}}+\mathrm{fd}\right)^{\wedge} 2$. The smooth term ti(logage) refers to an unknown linear combination of splines derived from logage, and the tensor product term ti(logage, birth) allows for interaction of logage with birth. In each smooth, the basis dimension is set at 10. By default, "cubic regression splines" are used with the ti terms. The smoothing parameter optimisation method indicated by "ML" is Marginal Likelihood. For details see [21] and ?gam and ?ti (within mgcv). Setting family $=$ "quasipoisson $"$ allows a mean-variance relation $\mathrm{V}=\mu \phi$ where $\mathrm{V}$ is the variance about mean $\mu$ and $\phi>0$ is a scale factor. For the Poisson family, $\phi=1$, but any positive scale can be set, or estimated as below.

The dose covariate is highly skewed and, in the grouped data, multimodal. Over $70 \%$ of py in the full data has dose $<20 \mathrm{mGy}$, or over $60 \%$ if NIC cells are excluded. Likewise over $84 \%$ has dose $<100 \mathrm{mGy}$, or $79 \%$ if NIC cells are 
excluded. The grouped data cells are also stratified by dose category, an analytical decision embodied in the anonymous public data. For example, each cell in $\mathrm{A}^{-}$or $\mathrm{A}^{+}$will contain records with dose either below $5 \mathrm{mGy}$ or between 5 and 20 mGy.

To adjust for this irregular distribution, when dose $=\mathrm{D}$ define ecdos as the proportion of cells with dose $\leq \mathrm{D}$, a monotone increasing function of dose with an approximately uniform distribution. It increases quickly where dose is concentrated, remaining static where dose is sparse. While $\max ($ ecdos $)=1, \min ($ ecdos $)>0$ due to cells with dose $=0$. Formally, ecdos is the empirical cumulative distribution function of dose, obtained in $R$ as ecdf(dose)(dose), with inverse transformation quantile(dose,). Figure 1 shows ecdos and dose in $\mathrm{B}^{+}$.

The initial model P0 (and its quasipoisson variant Q0) are enlarged with terms using ecdos or dose and interactions, as shown in Table 2.

The nested chains P0 $\subset$ P2e $\subset$ P3e $\subset$ P5ae, P0 $\subset$ P2e $\subset$ P4ae $\subset$ P5ae, P0 $\subset$ P2e $\subset$ P3e $\subset$ P5se, and P0 $\subset$ P2e $\subset$ P4se $\subset$ P5se give models of increasing complexity using ecdos with agex or since respectively. Corresponding nested chains P0 $\subset$ P2d etc. are defined using dose instead of ecdos. P2 refers to the pair P2e and P2d, etc.

The scale for a quasipoisson model $\mathrm{M}$ is estimated iteratively via Pearson $\chi^{2}=\sum(o b s-f i t)^{2} / f i t$, where fit is the fitted value, by seeking $\hat{\phi} \simeq \chi^{2} / d$ fres where dfres is the residual degrees of freedom (\# of records - effective degrees of freedom).

Additional file 1: Appendix A describes methods for Relative Risk RR, confidence intervals based on the Bayesian posterior distribution of $\beta$, dose response curves, and model selection. Simulation tests used to choose the preferred method of smoothing parameter optimisation and to validate the models are outlined in Additional file 1: Appendix B.

Numerical results are given at 2.5 and $10 \mathrm{mGy}$ in $\mathrm{A}^{+-}$, $2.5,10,20,40,60,80 \mathrm{mGy}$ in $\mathrm{B}^{+-}$, and at 2.5, 10, 100, 200, 300, and $400 \mathrm{mGy}$ in $\mathrm{C}^{+-}$. Confidence intervals are 90\% (lower and upper 95\% confidence limits LCL95\% and UCL95\%) unless otherwise specified, and "borderline" denotes $0.98 \leq$ LCL95\% $\leq 1$.
Initially, no minimum value of the smoothing parameters was imposed. However, if the visual output was "too wiggly", indicating overfitting, min.sp was increased until the output appeared fairly smooth, a subjective judgement.

In each data cell, a fitted model gives predicted values $\mu$, with $\sum \mu=\sum o b s$, and predicts values $\mu_{0}$ if dose had been reset to 0 , or ecdos reset to ecdf(dose)(0). The attributable fraction $1-\sum \mu_{0} / \sum \mu$ is the predicted number of additional cases above the baseline, as a proportion of those observed. $\sum \mu / \sum \mu_{0}$ estimates the Standardized Incidence Ratio from the fitted model.

The age distribution of cases varies between cancer sites. Define the "mid" age (and agex, since) for a site and dose range as that below which half the cases occur. Table 1 includes the mid values by sites in $\mathrm{B}^{+}$; values for other dose ranges are similar. Results are reported at the mid age rounded to the nearest 5 years, and where appropriate at the rounded mid agex or since.

For a fitted model $M$, set $I_{M}=1$ on any data cell for which LCL95\% > 1, and 0 otherwise. The proportion of obs (or py) for which $I_{M}=1$ measures the extent to which $R R>1$ is significant across the dose range. Since $I_{M}=$ 0 if dose $=0$, the proportions are obtained for $D_{0}$, those cells with dose $>0$, or for $\mathrm{D}_{0.5}$ (dose $>0.5 \mathrm{mGy}$ ). Corresponding proportions are obtained on $\mathrm{S}_{0.5}$ (since $<35$ and dose $>0.5 \mathrm{mGy}$ ), or $\mathrm{T}_{0.5}$ (agex $>35$ and dose $>0.5 \mathrm{mGy}$ ), or $\mathrm{S}_{0.5} \cap \mathrm{T}_{0.5}$. Likewise, the indicator $\mathrm{J}_{\mathrm{M}}=1$ on any data cell for which UCL95\% $<1$, and 0 otherwise. The proportion of obs (or py) for which $\mathrm{J}_{M}=1$ measures the extent to which $R R<1$ is significant across the dose range.

Selected models for all solid cancers were re-fitted after excluding exceptional data points as detected by Cook's Distance, or with thin plate regression splines, or for urban residents, or with RBE as obtained in [25].

For a fitted model $M$, influence $(M)$ is a vector of leverage at each cell, and plotting influence(M1)/influence(M2) against dose compares model sensitivities in different dose regions. The ability of ecdos and dose models to detect hypothetical dose-responses was compared by simulation (Appendix B). Further simulations considered the uncertainty in dose.
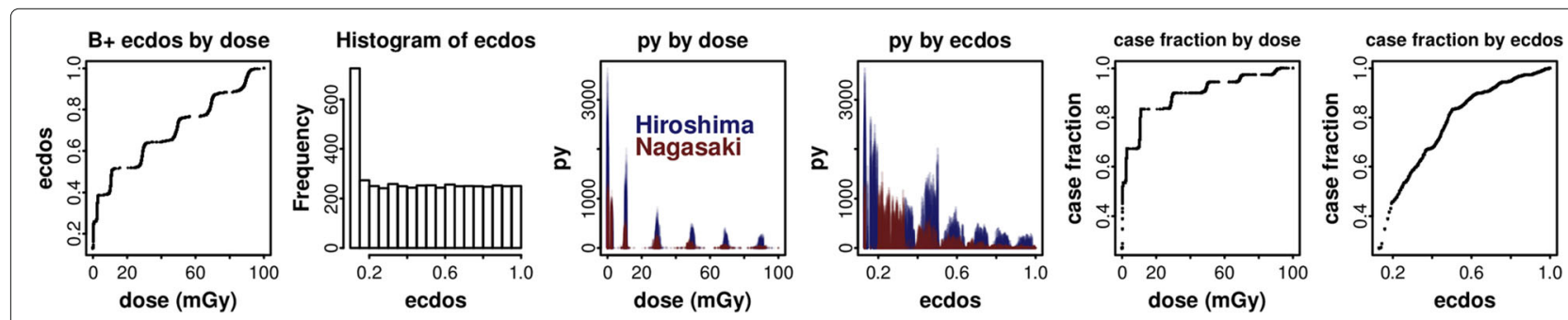

Fig. 1 Empirical cumulative distribution of dose. The ecdf of dose, denoted ecdos, is plotted against dose in $\mathrm{B}^{+}(0-100 \mathrm{mGy}$ including Not in City). The histogram is approximately uniform. py and case fractions are plotted against dose and ecdos 
Table 2 Model definitions

\begin{tabular}{|c|c|c|c|c|c|c|}
\hline Model & Family & $\phi$ & Model & Family & $\phi$ & Terms \\
\hline PO & poisson & 1 & Q0 & quasipoisson & $>0$ & $\operatorname{offset}(\log ($ py $))+(f d+f c)^{\wedge} 2+$ ti (logage $)+t($ birth $)+$ ti(logage,birth) \\
\hline P1e & poisson & 1 & Q1e & quasipoisson & $>0$ & tecdos \\
\hline P2e & poisson & 1 & Q2e & quasipoisson & $>0$ &.$+\mathrm{ti}(\mathrm{ecdos})$ \\
\hline P3e & poisson & 1 & Q3e & quasipoisson & $>0$ &.$+\mathrm{ti}($ ecdos)+ti(ecdos,logage) \\
\hline P4ae & poisson & 1 & Q4ae & quasipoisson & $>0$ &.$+\mathrm{ti}($ ecdos)+ti(ecdos,agex) \\
\hline P4se & poisson & 1 & Q4se & quasipoisson & $>0$ &.$+\mathrm{ti}($ ecdos) $+\mathrm{ti}($ ecdos,since) \\
\hline P5ae & poisson & 1 & Q5ae & quasipoisson & $>0$ &.$+\mathrm{ti}($ ecdos)+ti(ecdos,logage) +ti(agex)+ti(ecdos,agex) \\
\hline P5se & poisson & 1 & Q5se & quasipoisson & $>0$ &.+ ti(ecdos)+ti(ecdos,logage) +ti(since)+ti(ecdos,since) \\
\hline P1d & poisson & 1 & Q1d & quasipoisson & $>0$ & +dose \\
\hline$P 2 d$ & poisson & 1 & Q2d & quasipoisson & $>0$ & +ti(dose) \\
\hline P3d & poisson & 1 & Q3d & quasipoisson & $>0$ &.+ ti(dose)+ti(dose,logage) \\
\hline P4ad & poisson & 1 & Q4ad & quasipoisson & $>0$ & $.+\mathrm{ti}($ dose $)+\mathrm{ti}($ dose,agex $)$ \\
\hline P4sd & poisson & 1 & Q4sd & quasipoisson & $>0$ & $.+\mathrm{ti}($ dose $)+\mathrm{ti}($ dose, since) \\
\hline P5ad & poisson & 1 & Q5ad & quasipoisson & $>0$ &.+ ti(dose)+ti(dose,logage) +ti(agex)+ti(dose,agex) \\
\hline P5sd & poisson & 1 & Q5sd & quasipoisson & $>0$ & $.+\mathrm{ti}($ dose $)+\mathrm{ti}($ dose,logage $)+\mathrm{ti}($ since $)+\mathrm{ti}($ dose, since) \\
\hline
\end{tabular}

Models use cubic regression (cr) splines, with basis dimension 10

Model fitting by method $\mathrm{ML}$

".+" : augment the initial model PO (or Q0) by the terms which follow

ti refers to a smooth term on the enclosed covariate(s)

These intensive computations were applied only for all solid cancers. Elsewhere, the Bayesian posterior CIs (Appendix A) are reported without further validation. Some codes with commentary are shown as additional files.

\section{Results}

For all solid cancers, no minimum smoothing parameters were imposed. Basis dimension $\mathrm{k}=10$ was sufficient when tested by gam.check or modelling residuals against covariates. The ML method of smoothing parameter optimisation was preferred to alternatives, giving 95\% CIs which performed well under simulation tests described in Appendix B and shown in Fig. 2. ML is discussed in [24].

P5ae, P5se, P5ad and P5sd were cross-validated (Appendix B) in $\mathrm{A}^{+-}$, to test the proportion of deviance explained and the estimated RR $\left.\right|_{10}$ mGy with $90 \%$ CIs, see Additional file 1: Table S1.

Additional file 1: Figure S1 shows the dose response in $\mathrm{B}^{+}$. All plots show RR significantly raised, although the response is lower with the dose models. Plots with REML or GCV.Cp optimisation methods are similar. For the ecdos models, the appearance of the response reflects the relation between ecdos and dose (see Fig. 1); corresponding curves of RR against ecdos are regular.

Figure 3 compares the responses of P5se and P $5 \mathrm{sd}$ in $\mathrm{A}^{+}$, $\mathrm{B}^{+}$and $\mathrm{C}^{+}$, evaluated at age 70 and since 35 . Curves are similar over $\mathrm{A}^{-}, \mathrm{B}^{-}$and $\mathrm{C}^{-}$, and for P5ae and P5ad at age 70 and agex 35 , with slightly weakened significance.
Data simulated over $\mathrm{B}^{-}$from a variety of hypothetical forms of the true dose-response curve, without interactions, was fitted with P2e and P2d (Appendix B). Table 3 has results for 10 curves, comparing models by the mean coverage of their respective CIs across the dose range. Models with ecdos give better or equivalent coverage, unless the true dose-response is linear.

Figure 4 displays the simulated geometric mean fitted values and CIs for two of these curves ( $\mathrm{R} 1$ and $\mathrm{C} 2$ ), showing that the dose model underestimates the true response while the ecdos model captures it correctly.

The increased sensitivity of ecdos models at low doses is seen in Additional file 1: Figure S2, plotting the ratio of influence with P5sd to that with P5se, against dose. In $\mathrm{B}^{+-}$ and $\mathrm{C}^{+-}$low doses have less influence on P5sd, reducing its sensitivity to their effects.

Splines constructed from ecdos have better variability than dose splines at very low doses, lowering the internal baseline estimates. Additional file 1: Figure S3 compares splines from P2d, P2e, and P2g constructed from gdos, a smooth approximation to ecdos, when modelling over $\mathrm{B}^{+}$. Although the P2g response curve is simpler, the parameters of gdos vary with dose range and its distribution is multimodal, whereas ecdos has a universal definition and an approximately uniform distribution.

Interactions in $\mathrm{B}^{+}$are shown by perspective plots in Fig. 5, fitting P5a and P5s with either ecdos or dose, and fixing age $=70$ or agex $=35$ or since $=35$. Figure 6 shows $\left.\mathrm{RR}\right|_{10 \mathrm{mGy}}$ and $90 \%$ CIs at age 70, using P5a and P5s fitted 


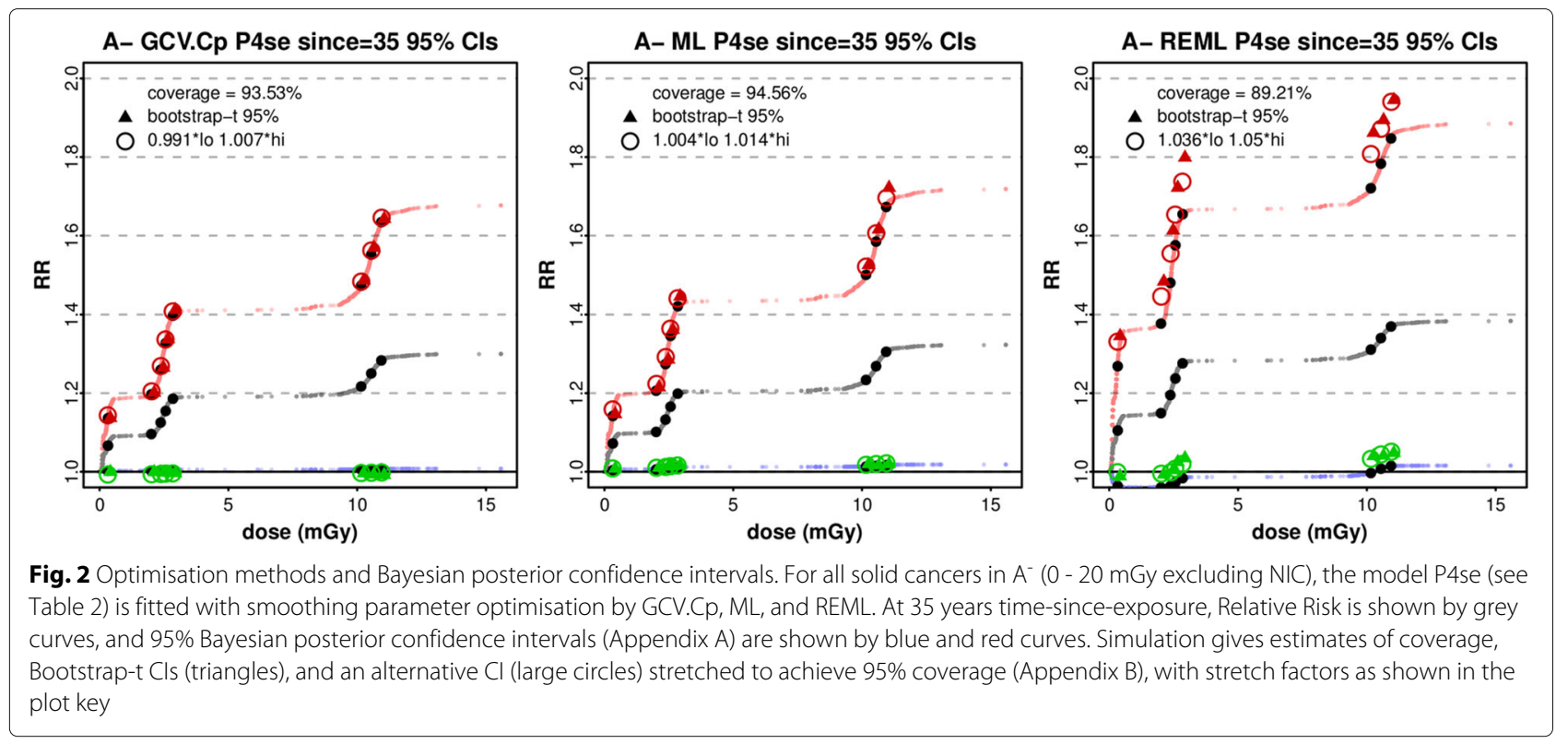

over $\mathrm{A}^{+}, \mathrm{B}^{+}$and $\mathrm{C}^{+}$. With P5a at age $70 \mathrm{RR}$ increases with agex at all doses in $\mathrm{A}^{+-}, \mathrm{B}^{+-}$or $\mathrm{C}^{+-}$. With $\mathrm{P} 5 \mathrm{~s}$ at age 70 , $\mathrm{RR}$ decreases (or is constant) with since at all doses. The

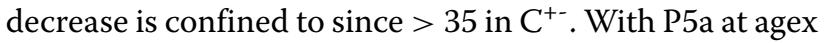
35 RR generally decreases (or is constant) with age. With $\mathrm{P} 5 \mathrm{~s}$ at since $35 \mathrm{RR}$ increases (or is constant) with age in $\mathrm{A}^{+-}$and $\mathrm{B}^{+-}$, but decreases with age at higher doses in $\mathrm{C}^{+-}$.

Poisson model selection methods (Appendix A) were applied to choose preferred models. P5se is preferred in all six dose ranges by method 1 (lowest ML score). The three methods chose models with ecdos in 17 of 18 cases. Additional file 1: Table S2 shows the preferred Poisson models for each range and selection method. As appropriate, RR is shown at age 70 , agex 35 , or since 35 . All preferred models show RR elevated at 2.5, 10, 20, 40,60,80, 100, 200, 300, and $400 \mathrm{mGy}$, with LCL95\% > 1, except for method 2 in $\mathrm{B}^{+}$where P3d is preferred, with $\mathrm{LCL} 95 \% \geq 1$. In all cases Deviance $<$ dfres. The proportion of Deviance explained
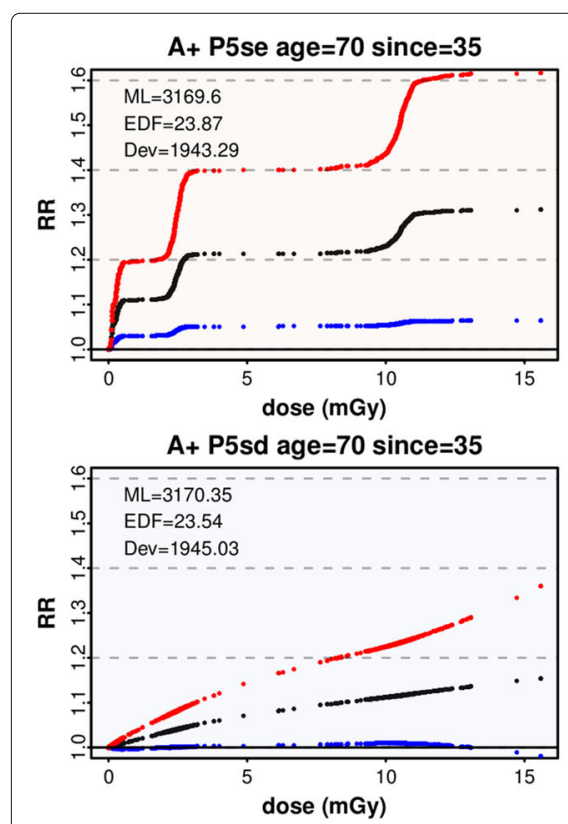

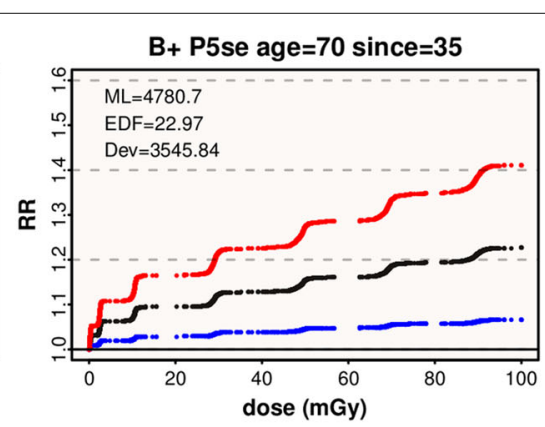

$B+P 5 s d$ age $=70$ since $=35$

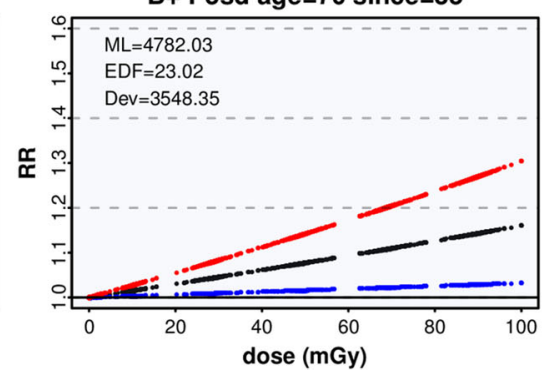

C+ P5se age $=70$ since $=35$

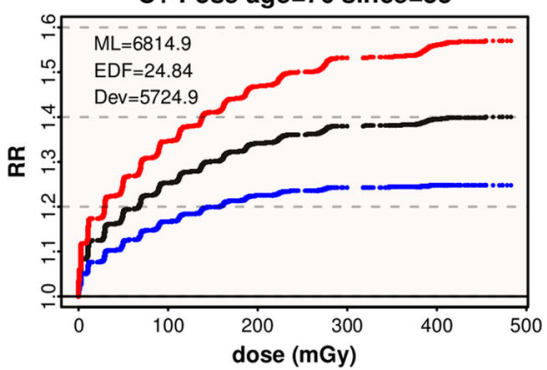

C+ P5sd age $=70$ since $=35$

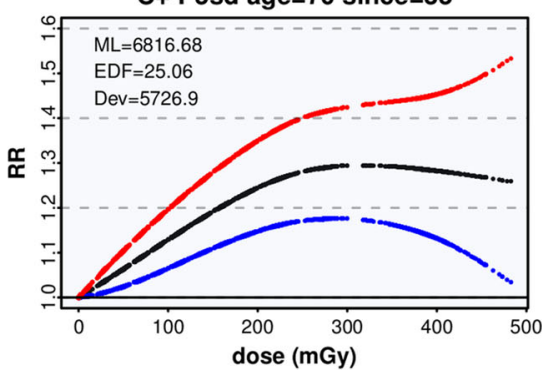

Fig. 3 Dose response of preferred models in $\mathrm{A}^{+}, \mathrm{B}^{+}, \mathrm{C}^{+}$. For all solid cancers, the top row shows the Poisson model P5se selected in each dose range to minimise ML, with the corresponding dose model P5sd on the bottom row. RR and $90 \% \mathrm{Cls}$ at the rounded mid since and age, are plotted against dose. ML scores, effective degrees of freedom, and Deviance are listed. For model definitions see Table 2 
Table 3 Coverage by P2e and P2d of true dose response

\begin{tabular}{|c|c|c|c|c|c|c|c|c|}
\hline Name & ERR & $\beta$ & $\sigma$ & $\tau$ & $\gamma$ & Form & Mean (lhe) & Mean (Ihd) \\
\hline N1 & $g(d, \beta, \sigma, \tau)$ & 0 & 0 & & & null & $95.18 \%$ & $94.90 \%$ \\
\hline L1 & $g(d, \beta, \sigma, \tau)$ & 0.002 & 0 & & & linear & $81.46 \%$ & $94.56 \%$ \\
\hline $\mathrm{H} 1$ & $f(d, \beta, \sigma, \tau)$ & 0.004 & -0.2 & 0.05 & & hormesis & $75.08 \%$ & $69.86 \%$ \\
\hline P1 & $f(d, \beta, \sigma, \tau)$ & 0 & 0.2 & 0.05 & & plateau & $93.36 \%$ & $69.68 \%$ \\
\hline C1 & $f(d, \beta, \sigma, \tau)$ & 0.0012 & 0.08 & 0.5 & & two phase & $90.88 \%$ & $50.08 \%$ \\
\hline C2 & $f(d, \beta, \sigma, \tau)$ & 0.015 & 0.75 & 0.2 & & two phase & $94.80 \%$ & $13.98 \%$ \\
\hline R1 & $g(d, \beta, \sigma, \tau)$ & 0.002 & 0.01 & 0.08 & & two phase & $93.64 \%$ & $68.14 \%$ \\
\hline R2 & $g(d, \beta, \sigma, \tau)$ & 0.002 & 0.01 & 0.04 & & two phase & $93.98 \%$ & $66.10 \%$ \\
\hline T1 & $h(d, \gamma)$ & & & & 50 & threshold & $88.40 \%$ & $87.66 \%$ \\
\hline T2 & $h(d, \gamma)$ & & & & 25 & threshold & $86.88 \%$ & $86.90 \%$ \\
\hline
\end{tabular}

All Solid cancers, fitting over $B^{-}$

ERR: true value as specified by function

$f(d, \beta, \sigma, \tau)=\beta \cdot d+\sigma \cdot(1-\exp (-\tau \cdot d))$

$g(d, \beta, \sigma, \tau)=\beta \cdot d+\sigma \cdot d \cdot \exp (-\tau \cdot d)$

$h(d, \gamma)=$ ifelse $\left(d<\gamma, 0,1 / 5 \cdot((d-\gamma) /(100-\gamma))^{3}\right)$

Mean (Ihe or Ihd): mean coverage at 10,20...100 mGy of 95\% CI for P2e (P2d) see also Appendix B

varies from $46.1 \%$ in $\mathrm{C}^{-}$to $68.1 \%$ in $\mathrm{A}^{+}$. The attributable fraction varies from $1.4 \%$ (method 2 in $\mathrm{B}^{+}$) to $9.4 \%$ in $\mathrm{A}^{-}$.

Additional file 1: Table $\mathrm{S} 2$ also shows the percentage of cases and py in $\mathrm{D}_{0}$ for which RR $>1$, LCL95\% $>1$, or UCL95\% < 1 ; likewise for cells in $\mathrm{D}_{0.5}, \mathrm{~S}_{0.5}, \mathrm{~T}_{0.5}$, or $\mathrm{S}_{0.5}$ $\cap \mathrm{T}_{0.5}$ (see "Methods"). For each of these preferred models, cell and dose ranges, RR $>1$ holds for over $80 \%$ of cases and py, and UCL95\% < 1 never occurs. Except with method 2 in $\mathrm{B}^{+}$, LCL95\% > 1 holds for over $70 \%$ of cases in $\mathrm{T}_{0.5}$, over $75 \%$ of cases in $\mathrm{S}_{0.5}$, and over $90 \%$ of cases in $\mathrm{S}_{0.5} \cap \mathrm{T}_{0.5}$.

Significant elevation of RR persists over a wide range of age and since (or agex), as shown for all models in $\mathrm{B}^{+}$in Fig. 7. With the ecdos models, RR is significantly raised for regions containing age $>55$, agex $>35$, or since $<35$. With the dose models, the regions of significance are similar, though smaller. For the preferred (method 1) Poisson models in $\mathrm{A}^{+}, \mathrm{B}^{+}$, and $\mathrm{C}^{+}$, Fig. 8 shows the dose response, the dependence of $\mathrm{RR}$ on since and age, and the regions of significance.

The dose models themselves give non-linear responses over $\mathrm{C}^{+}$(see Fig. 3). With P2d, RR rises approximately linearly below $150 \mathrm{mGy}$, before tapering. Anova comparison with $\mathrm{P} 1 \mathrm{~d}$, in which dose appears as a log-linear unsmoothed term, gives $\mathrm{p}=0.015$. Below $100 \mathrm{mGy}$, the Excess Relative Risk (ERR $=R R-1$ ) is about 70\% higher with P2d than with P1d. With P5sd RR tapers above $150 \mathrm{mGy}$ as with P2d. The term ti(since) has e.d.f. = 3.658 and $\mathrm{p}=0.037$, and anova $(\mathrm{P} 3 \mathrm{~d}, \mathrm{P} 5 \mathrm{sd})=0.008$ while anova $(\mathrm{P} 4 \mathrm{sd}, \mathrm{P} 5 \mathrm{sd})=0.07$. Thus P5sd is decidedly nonlinear in dose, whose interaction with logage is borderline, but since has significant impact.
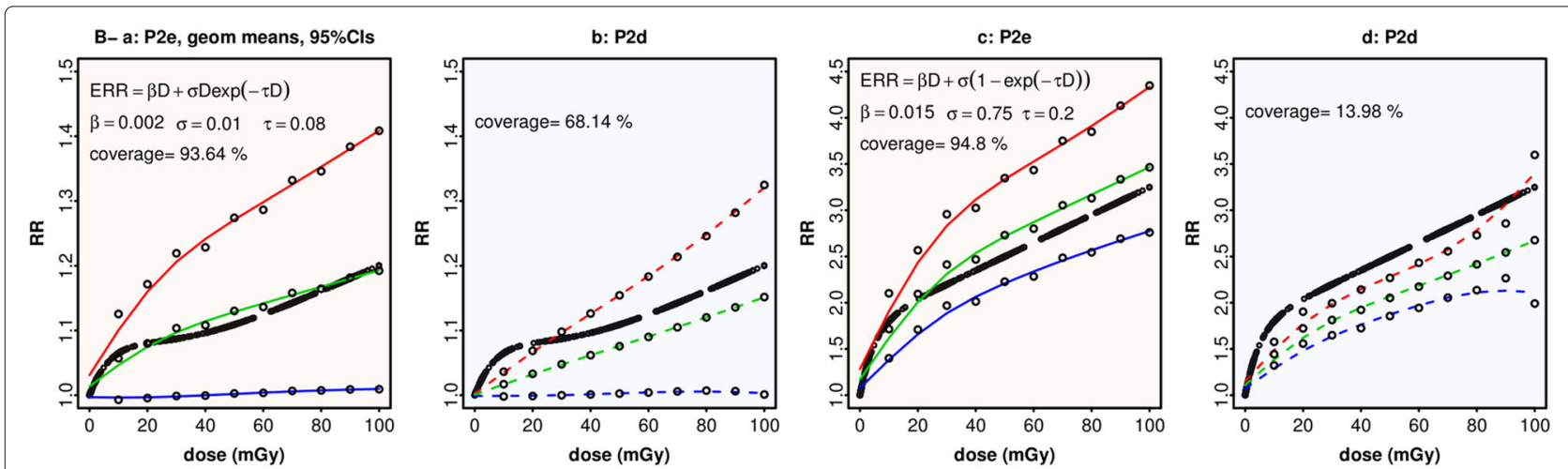

Fig. 4 Ability to capture a known response. Data is simulated (Appendix B) over $B^{-}$with RR $=1+$ ERR as specified by the formulas in Panels $\mathbf{a}$ and $\mathbf{c}$, without interactions. Thick black curves show the true RR. Simulated data is modelled by P2e (Table 2) in Panels $\mathbf{a}$ and $\mathbf{c}$, and remodelled by P2d (Table 2) in Panels $\mathbf{b}$ and d. At dose $=10,20 \ldots 100 \mathrm{mGy}$, fitted values and $90 \% \mathrm{Cls}$ are obtained at each simulation step. Coverage is the $\%$ over all 10 doses for which the simulated $\mathrm{Cl}$ contains the true RR. Panels display the geometric means (hollow dots) interpolated by smoothing splines, green for fitted values, blue (red) for lower (upper) 95\% Cls 


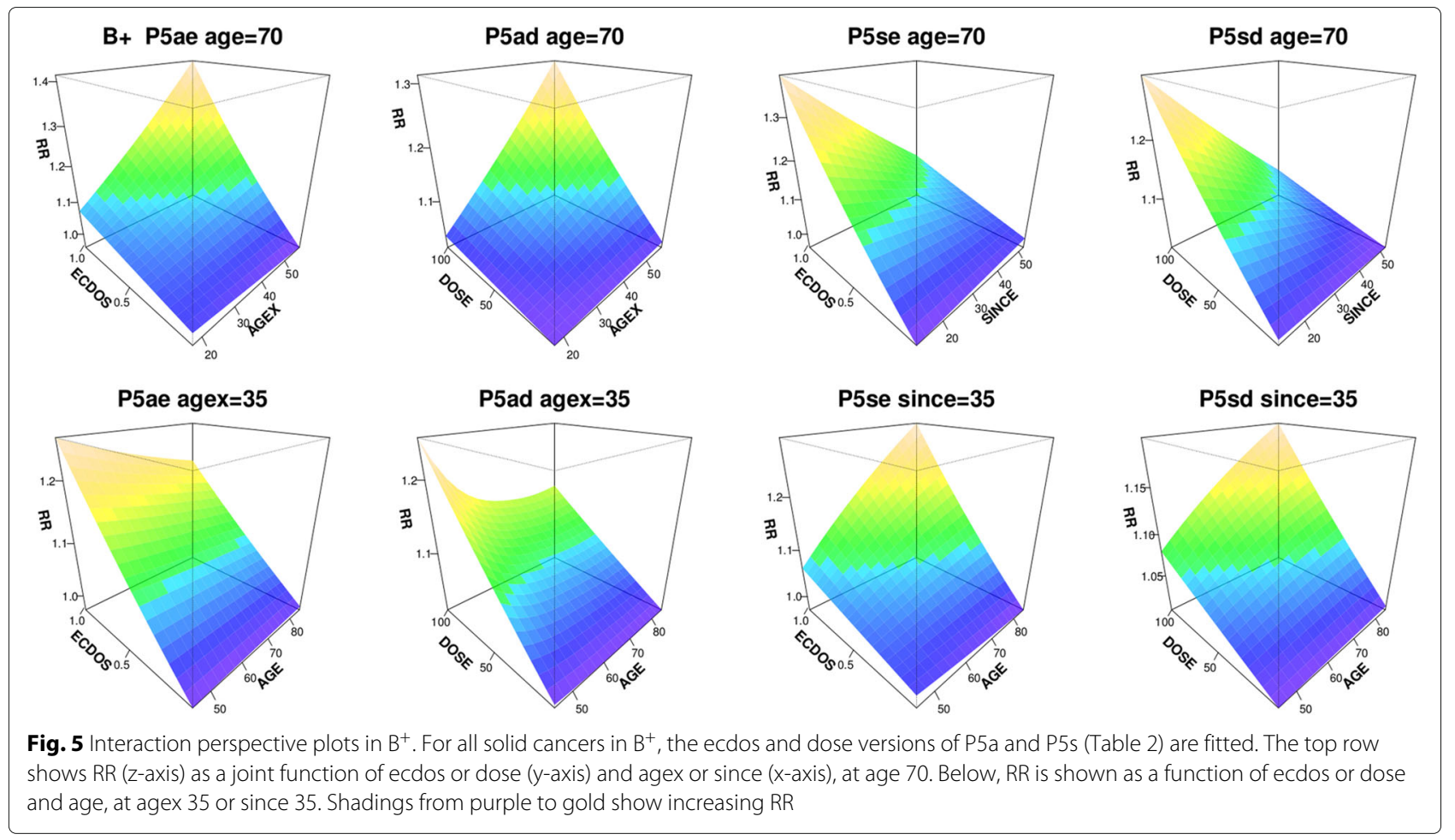

The dose models also show RR raised in $\mathrm{A}^{+-}$. For P1d and $\mathrm{P} 2 \mathrm{~d}$, in both ranges $\left.R R\right|_{10 \mathrm{mGy}}=1.09(0.99,1.19)$. P4sd is preferred amongst dose models by methods 2 and 3 . At since 35 , in $\left.A^{-} R R\right|_{10 ~ m G y}=1.11(1.00,1.22)$ with significant elevation when since $<35$. In $\left.A^{+} R R\right|_{10 ~ m G y}=1.09$ $(0.99,1.19)$ with significant elevation when since $<32$. In $\mathrm{A}^{+-}$P5sd is preferred amongst dose models by method 1 . At age 70 , since 35 in $\left.\mathrm{A}^{-} \mathrm{RR}\right|_{10 \mathrm{mGy}}=1.11(1.00,1.23)$ whilst in $\left.\mathrm{A}^{+} \mathrm{RR}\right|_{10 \mathrm{mGy}}=1.11(1.01,1.22)$ and $\mathrm{RR}$ is significantly raised for $37.2 \%$ (28.2\%) of cases (py) in $\mathrm{D}_{0.5}, 75.0 \%$ (42.3\%) in $\mathrm{S}_{0.5}$, and $68.4 \%(79.6 \%)$ in $\mathrm{T}_{0.5}$.
In general, point estimates and CIs are primarily affected by the dose range (less by the inclusion of NIC) and the choice of dose or ecdos.

The stability of these results was tested, first by using thin plate regression splines. RR and CIs were similar to those shown in Additional file 1: Table S2, but cubic regression splines gave lower ML scores. If exceptional cells detected visually by Cook's Distance (CD) are deleted, RR values and 90\% CIs were slightly altered from those in Additional file 1: Table S2, but still significantly raised (borderline for $\mathrm{B}^{+}$method 2). Deletion affected
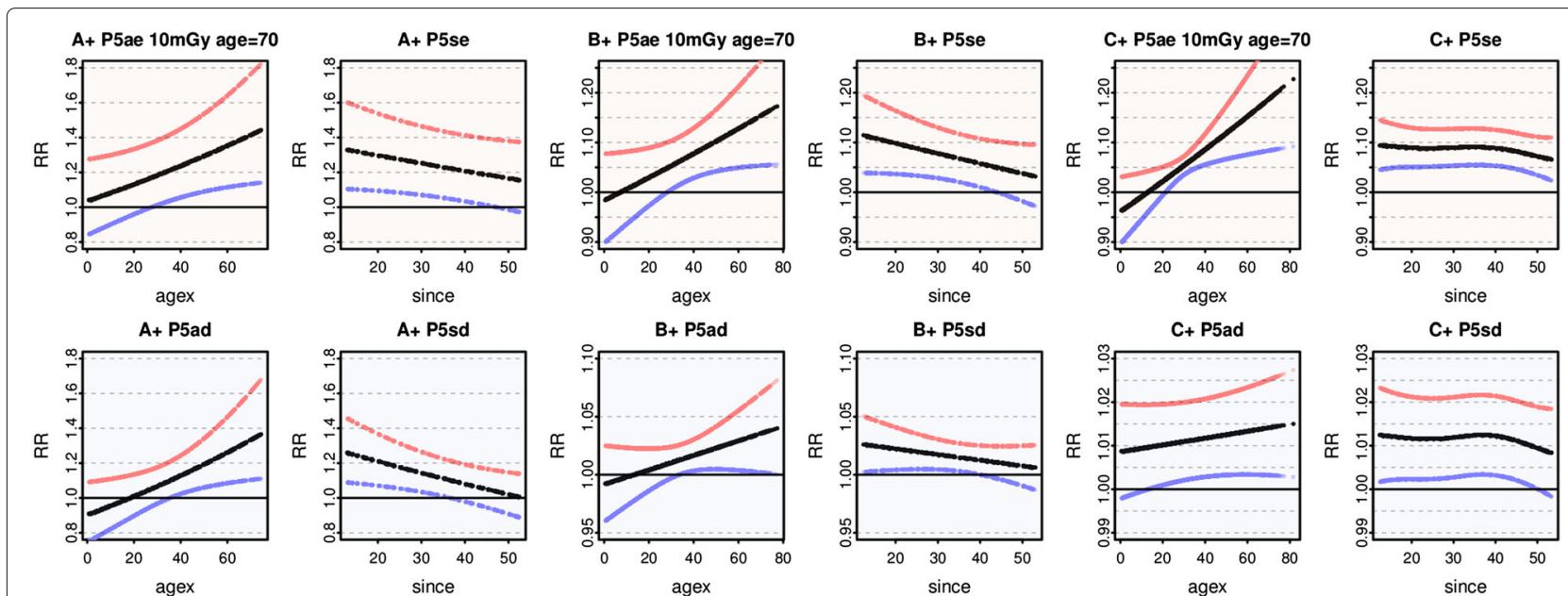

Fig. 6 Interactions at $10 \mathrm{mGy}$. For all solid cancers in $\mathrm{A}^{+}, \mathrm{B}^{+}$, and $\mathrm{C}^{+}, \mathrm{RR}$ and $90 \% \mathrm{Cl}$ at $10 \mathrm{mGy}$ is plotted against agex or since, at age 70 , for the ecdos and dose versions of P5a and P5s (Table 2). The top row shows ecdos models, with corresponding dose models below 


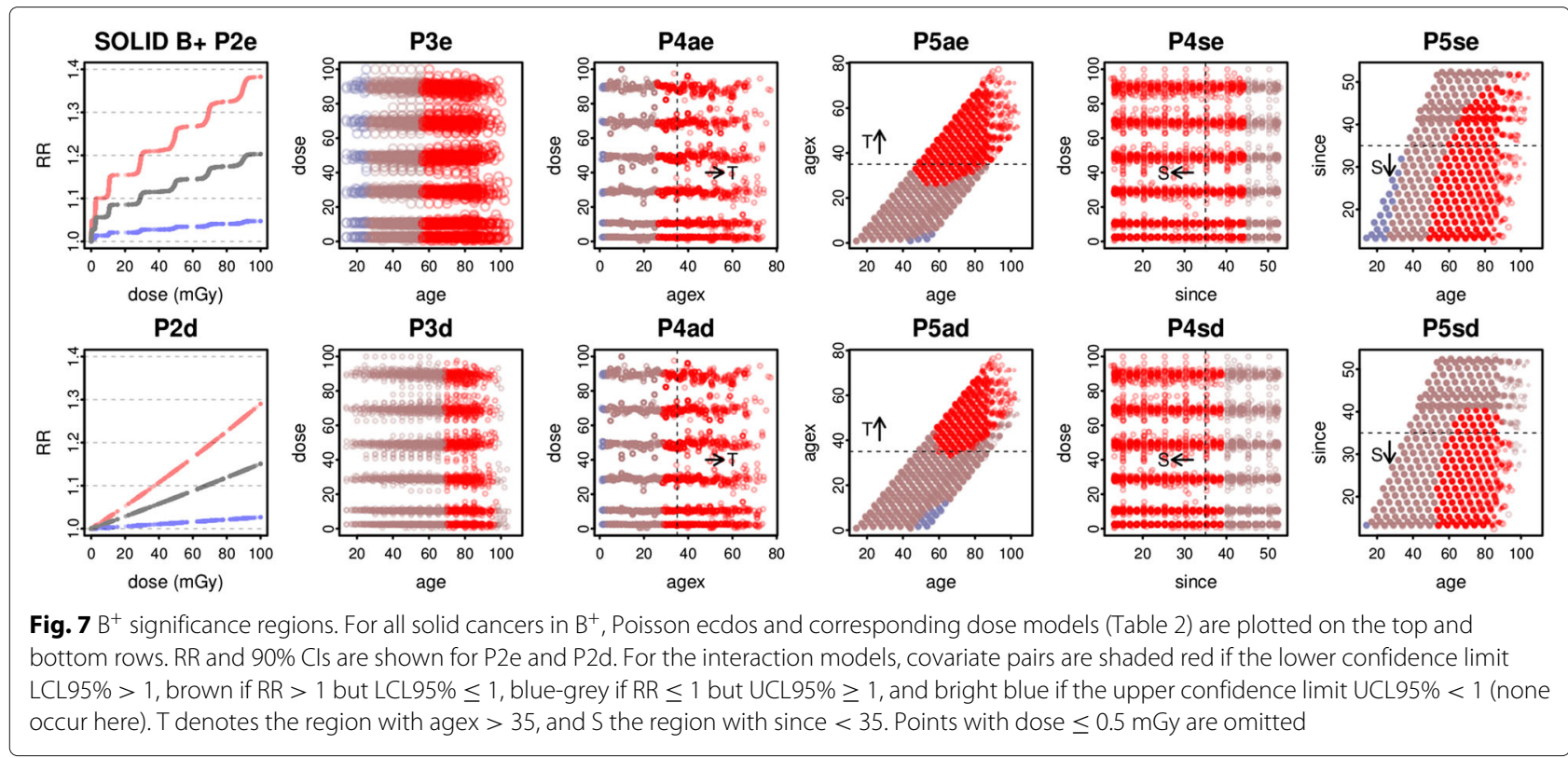

the choice of preferred models only for $\mathrm{C}^{-}$, where P5ae was preferred by all methods, and $\mathrm{C}^{+}$, where P5se was preferred by all methods. When preferred models were refitted after excluding cells with the highest $5 \%$ of $C D$ values, $R R$ was raised with at least borderline significance across each dose range.

The combined impact of sampling variability and lognormal dosimetry errors was simulated (see Appendix B) using P5se and P5sd in $\mathrm{A}^{-}$, shown in Additional file 1: Figure S4. Geometric mean fitted values and 90\% CIs are below the values derived from the original data, but still raised with borderline significance; while bootstrap-t [26] and stretched CIs derived from the simulated data remain significantly raised.

Models were fitted with RBE as the function of neutron dose $D_{n}$ shown in [25] (Fig. 4 and Eq. (2)). This raises

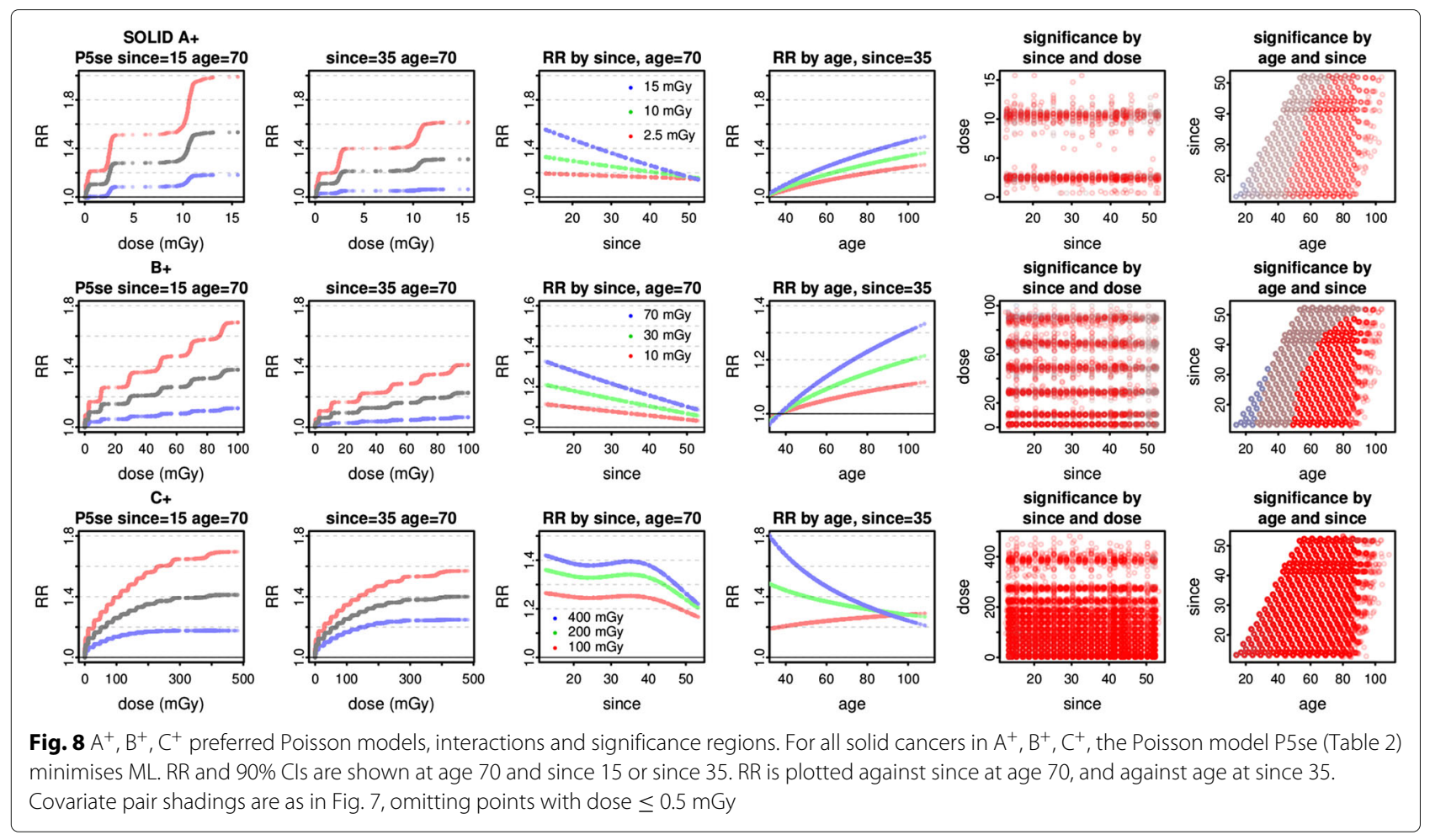


the dose assigned, as RBE $>50$ in the ranges considered here (for which $D_{n}<0.003$ Gy). Results are shown in Additional file 1: Table S3. In all dose ranges and with all three methods of Poisson selection, RR is elevated with at least borderline significance. In 15 out of 18 cases, and for 6 of 6 with method 1, the same models are selected, and $\mathrm{RR}$ and CIs are slightly lower but still comparable to those in Additional file 1: Table S2.

Excluding genital or, separately, respiratory cancers, the remaining solid cancers were modelled over $\mathrm{A}^{+}, \mathrm{B}^{+}$, and $\mathrm{C}^{+}$, with results in Additional file 1: Table S4. RR estimates are mainly similar to those in Additional file 1: Table S2. The heightened significance when agex $>35$ or since $<35$ generally survives these exclusions.

For urban residents in A, B, or C, the preferred Poisson models show RR elevated with at least borderline significance, and a tapering response at higher doses (see Additional file 1: Table S5). With method 3, RR is significantly elevated in each range.

In each dose range, the factor variables $\mathrm{fc}$ and $\mathrm{fd}$ have significant interaction, lowering the fitted values for rural Nagasaki residents. Modelling separately by city (Additional file 1: Table S6), for Hiroshima, models with since are preferred for every dose range and selection method. None show RR significantly or borderline raised at rounded mid age and since over $\mathrm{A}^{+-}$or $\mathrm{B}^{+-}$, but over $\mathrm{C}^{+-} \mathrm{RR}$ is significantly raised (method 1 ) or borderline (at 10 mGy, methods 2 and 3) at age 70 and since 35, and significantly raised (all methods) for high percentages of cases and py.

For Nagasaki, the preferred models show RR significantly raised in $\mathrm{A}^{+-}$and $\mathrm{B}^{-}$(and for method 1 in $\mathrm{B}^{+}$) at rounded mid age, since, or agex, and for high percentages of cases and py. Similar results are obtained over $\mathrm{C}^{+}$ with method 1, though exposure at younger ages appears more significant. Figure 9 shows the response by city at the respective rounded mid age and since or agex for the preferred models (method 1 ) in $\mathrm{A}^{+}, \mathrm{B}^{+}$, and $\mathrm{C}^{+}$, and the variation of significance with covariates.

Specific sites were analyzed by quasipoisson models with the Pearson estimate of scale $\hat{\phi}$. Additional file 1 : Table S7 gives details for $\mathrm{Q} 2$ in $\mathrm{B}^{+}$at 21 sites, using scale estimates from Q2e and Q2d with identical values of min.sp. The ML score of Q2d at $\hat{\phi}_{\mathrm{Q} 2 \mathrm{~d}}$ is compared with that of $\mathrm{Q} 2 \mathrm{e}$ refitted at $\hat{\phi}_{\mathrm{Q} 2 \mathrm{~d}}$, and vice-versa. The same covariate was preferred whichever optimised scale was used. The Q2 response curves and preferred covariates are shown in Fig. 10. Q2e curves are smoothed for display.

Several sites, including the uterus, show non-linear responses. For all solid, genital, respiratory, gallbladder, thyroid, and cns, the response is significantly elevated for both models in all cells in $\mathrm{D}_{0}$. With the preferred covariate, $R R$ is also significantly elevated throughout $D_{0}$ for the uterus and corpus, with weaker evidence for squamous, cervix, and rectum. For lung cancers, using the preferred covariate RR is elevated with borderline significance for over $80 \%$ of py in $\mathrm{D}_{0}$.

These 12 sites, along with the breast and liver which are radiosensitive at higher doses but show (non-significant) negative response in Fig. 10, were analysed with interaction models. In Additional file 1: Table S8, method 4 is used for each site and dose range, to choose the preferred quasipoisson model. Poisson selection by methods 1,2 , and 3 is also shown. Using the preferred quasipoisson models, RR and $90 \%$ CIs are shown along with the percentages of cases and py in $\mathrm{D}_{0}, \mathrm{D}_{0.5}, \mathrm{~S}_{0.5}, \mathrm{~T}_{0.5}$, and $\mathrm{S}_{0.5}$ $\cap \mathrm{T}_{0.5}$ for which $R R$ is elevated, significantly elevated, or significantly lowered.

$R R$ is significantly elevated across the dose range for all solid cancers in $\mathrm{A}^{-}, \mathrm{A}^{+}$(borderline), $\mathrm{B}^{+-}$, and $\mathrm{C}^{+-}$. Squamous cancers show significantly elevated $R R$ in $A^{-}, B^{-}$, $\mathrm{B}^{+}$(borderline $80 \mathrm{mGy}$ ), and $\mathrm{C}^{+-}$. The corpus has significant, highly elevated $R R$ in $A^{+-}$and $B^{+-}$. Thyroid $R R$ is significantly elevated in $\mathrm{A}^{+-}, \mathrm{B}^{+-}$, and $\mathrm{C}^{+-}$.

Respiratory RR is significantly lowered for $37.2 \%$ of py in $\mathrm{A}^{-}$(all with agex $<18$ ), $13.7 \%$ of py in $\mathrm{A}^{+}$(all with since $>44$ ) and $11.9 \%$ of py in $\mathrm{C}^{-}$(all with agex $<8$ ); it is significantly raised in $\mathrm{S}_{0.5}$ and $\mathrm{T}_{0.5}$ in $\mathrm{B}^{+-}$, and in $\mathrm{D}_{0.5}$, $\mathrm{S}_{0.5}$ and $\mathrm{T}_{0.5}$ in $\mathrm{C}^{+-}$. For the breast, $\mathrm{RR}$ is significantly lowered only for $10.9 \%$ of py in $\mathrm{B}^{-}$. There is little significant elevation in $\mathrm{A}^{+-}$or $\mathrm{B}^{+-}$, but $\mathrm{RR}$ is significantly raised in $\mathrm{D}_{0.5}, \mathrm{~S}_{0.5}$ and $\mathrm{T}_{0.5}$ in $\mathrm{C}^{-}$, and for all py in $\mathrm{C}^{+}$. For the liver, $\mathrm{RR}$ is significantly lowered for $24.8 \%$ of py in $\mathrm{A}^{-}$, $26.2 \%$ in $\mathrm{A}^{+}, 8.7 \%$ in $\mathrm{B}^{-}, 5.6 \%$ in $\mathrm{B}^{+}, 10.1 \%$ in $\mathrm{C}^{-}$, and $2.2 \%$ in $\mathrm{C}^{+}$.

Additional file 1: Figures S5-S10, for respiratory, uterus, and thyroid, correspond with Figs. 7 and 8 for all solid cancers. For the uterus, RR is a U-shaped function of age. For the thyroid, RR peaks around age 60 and decreases with agex.

Whilst the preferred models vary over the sites, dose ranges, and selection methods, ecdos models are chosen in 215 out of 336 cases, with a similar proportion for each selection method. Models with since are chosen in 194 cases and models with agex are preferred in 80. Among the 24 models for all solid cancers, ecdos is chosen in 22 , since in 18 , and agex only once. Overall, there is a clear preference for ecdos and since, and models with both covariates are preferred in 140 of 336 cases in total.

The male cohorts in $\mathrm{A}^{+}$and $\mathrm{B}^{+}$were fitted with Poisson models. In $\mathrm{A}^{+}, \mathrm{P} 5 \mathrm{ad}$ (method 1), P4ae (method 2) and P2e (method 3) are preferred. With P5ad at age 70 and with P4ae, the response increases with agex but lacks borderline significance. With P2e the response is insignificant. In $\mathrm{B}^{+}$, P5se (method 1), P4se (method 2$)$ and P2e (method 3) are preferred. With P5se at age 70, the response decreases with since but fails to reach borderline 


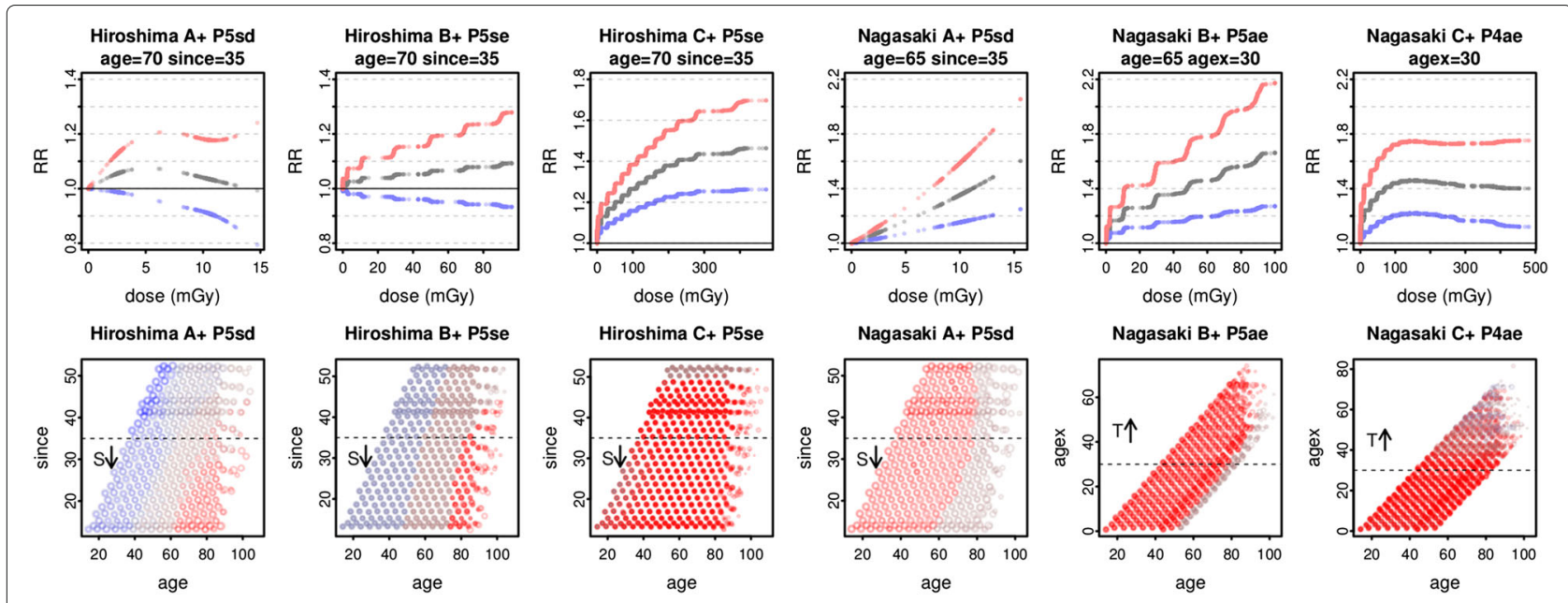

Fig. 9 Hiroshima and Nagasaki. For all solid cancers over $\mathrm{A}^{+}, \mathrm{B}^{+}, \mathrm{C}^{+}$, modelling is carried out separately by city, selecting the Poisson model to minimise ML in each case. In the top row, RR and $90 \%$ Cls are shown at the rounded mid age, agex or since as appropriate. In the bottom row, covariate pair shadings are as in Fig. 7, omitting points with dose $\leq 0.5 \mathrm{mGy}$

significance. With $\mathrm{P} 4 \mathrm{se}$, the response decreases with since and is borderline for since $\leq 20$. With P2e, the result is insignificant.

The combined cohorts in $\mathrm{A}^{+}$and $\mathrm{B}^{+}$were fitted with Poisson models, controlling for gender, city, distcat and their first order interactions, obtaining a gender averaged dose response. In $\mathrm{A}^{+}$, P5sd (method 1) and P4sd (methods 2 and 3) are preferred. For P4sd at 10 mGy RR is significantly raised $(90 \% \mathrm{CIs})$ when since $\leq 33$. At since
$30,\left.R R\right|_{10 \text { mGy }}=1.09(1.02,1.17)$. Results from P5sd at age 70 are nearly identical. In $\mathrm{B}^{+}$, P5se (method 1 ) and P4se (methods 2 and 3) are preferred. For P4se at since 30, $\left.R R\right|_{10 \mathrm{mGy}}=1.07(1.01,1.12)$, but significance is lost for $19 \leq$ since $\leq 27$.

\section{Discussion}

Low dose radiation has significant effects at cell level including some non-linear responses [27], but cancer

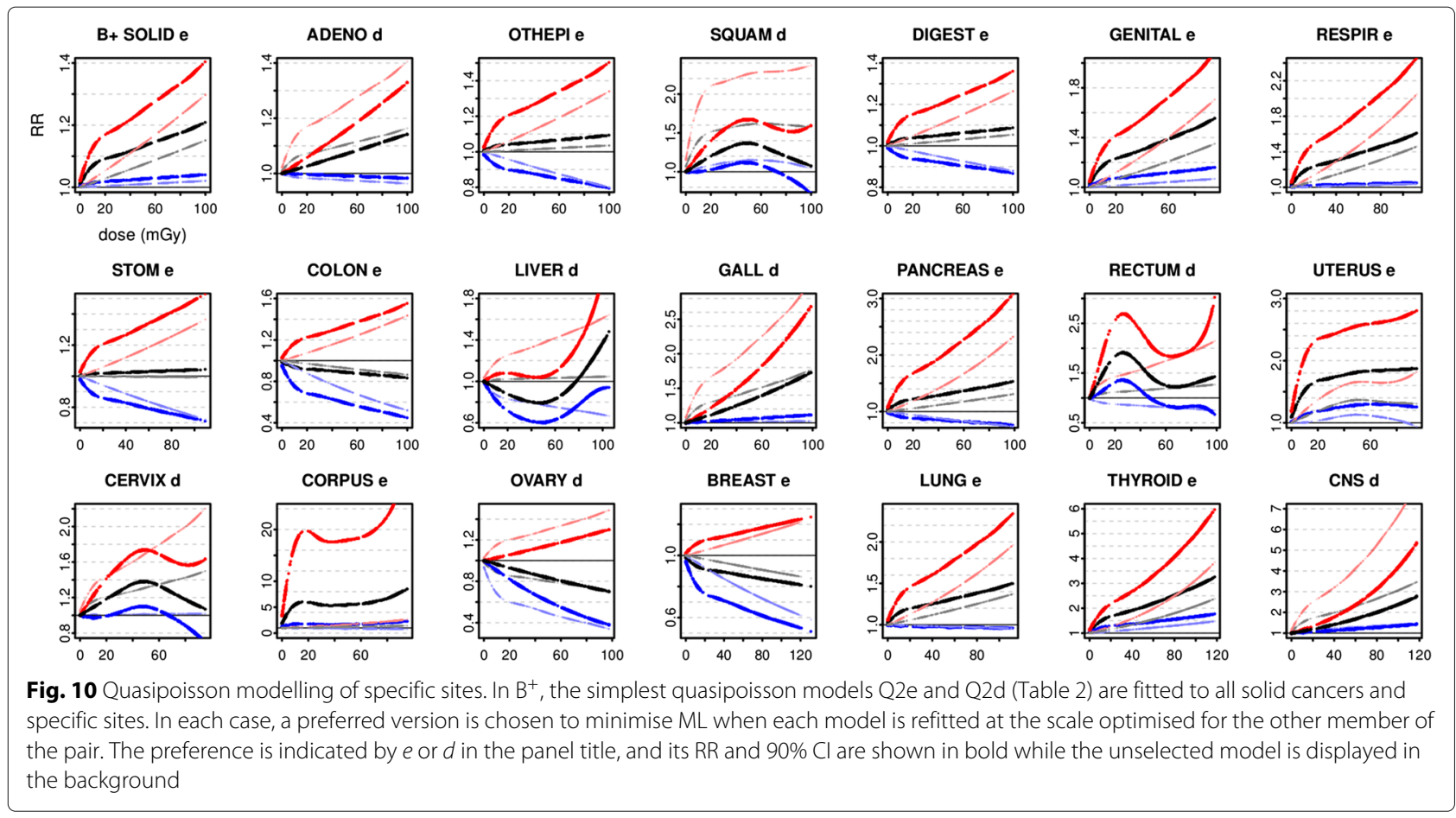


risk is typically modelled as a linear function of dose and the risk below $100 \mathrm{mGy}$ is still under debate. The body's multiple defences may filter out most abnormalities and the dose-response for disease could be linear.

In analysing the Japanese A-bomb survivors incidence or mortality, the Lifespan studies and other reports $[1,3,28,29]$ use a form of Poisson regression in which $\mathrm{ERR}=\mathrm{RR}-1$ is modelled as $\beta D$, where $\beta$ may be modified by sex, age and age-at-exposure through other parameters; alternatively, ERR $\sim \beta D+\gamma D^{2}$. If the response were known to take a specific form with the same parameter values at all doses, a model fitted to the full data could predict risk at $0.01 \mathrm{~Gy}$. Otherwise, a model which estimates risk at $1 \mathrm{~Gy}$ may be inappropriate at lower doses, as several studies recognise $[2,8,30]$.

In one approach to detecting non-linearity, numerical data can be reduced to categories before smoothing the results [1]. The outcome may depend on the cut points, and information is discarded by categorising data. The Generalized Additive Model $[19,21]$ is a more sophisticated method. Using GAM, covariates and interactions enter the model through unknown linear combinations of splines.

The public data released by RERF is stratified by essentially arbitrary dose categories with cutpoints $0,5,20$, $40 \mathrm{mGy} . .$. as well as categories of age and age-atexposure, city, sex and location. However the data also contains py-weighted mean values of dose, age, age-atexposure, and calendar year (giving time-since-exposure). The analysis uses these values, whose distribution, particularly for dose, is far from uniform. Stratification causes clumping of mean dose, but the individual exposures are also concentrated at low doses. As Preston observed [1] "One aspect of the LSS cohort that is often neglected is the highly skewed dose distribution and the large number of survivors who received low-dose, albeit at a high dose rate, exposure."

To compensate for stratification and skew, dose was converted to its empirical cumulative distribution, a monotone (invertible) transformation. The resulting variable ecdos is approximately uniformly distributed. Models using ecdos gave better fit for all solid cancers and many specific sites. This transformation may still be relevant with the individual data as dose remains skewed, though no longer clumped.

If the true dose-response is linear then modelling with dose is preferable. Whilst there is little difference in detecting a threshold, ecdos models are better able to capture alternatives, including plateau, hormesis, and a variety of curves with rapid initial rise and a smaller increase at higher doses. One such curve (Fig. 4 panels c and d) is loosely based on in vivo data for $\gamma$-H2AX Foci (see [31] (Fig. 1)).
The models have several sources of non-linearity, including the log link, the relation between ecdos and dose, and the splines. In fact, the fitted models are often log-linear in ecdos as a main effect. However, the interaction of ecdos or dose with logage and since or agex can introduce further non-linearities.

For all solid cancers, in $\mathrm{A}^{+-}, \mathrm{B}^{+-}$and $\mathrm{C}^{+-}$all three Poisson selection methods yield models with RR raised across the dose range at the rounded mid values of covariates, with at least borderline significance. Results are stable when deleting exceptional cells, simulating random errors in dosimetry, adjusting RBE, using different splines, or excluding genital or respiratory cancers. For all solid cancers, results are similar with Poisson or quasipoisson models, which explain around $68 \%$ of the deviance in $\mathrm{A}^{+}, 58 \%$ in $\mathrm{B}^{+}$, and $50 \%$ in $\mathrm{C}^{+}$.

For all solid cancers in $\mathrm{A}^{+-}$, all the dose or ecdos Poisson models show effects which are at least borderline $(0.98 \leq$ LCL95\% $\leq 1)$ or significant (LCL95\% $>1)$ at 2.5 and $10 \mathrm{mGy}$, at the rounded mid values of covariates. The preferred models with ecdos refine the results. Significance persists over a wide range of covariates.

With the Nagasaki data, the Poisson model chosen to minimise ML shows RR elevated across the cohort in $\mathrm{A}^{+-}, \mathrm{B}^{+-}$, and $\mathrm{C}^{+}$; and in $\mathrm{B}^{+-} \mathrm{ERR} \mid 10 \mathrm{mGy}$ is more than twice the value for the combined cohort. Using a very different approach, Sasaki [7] found an exceptional dose response for Nagasaki (male and female).

For all solid cancers, risk estimates are similar with or without the Not In City data. NIC cells are not distinguished by the urban/rural status of the individual members' normal residence, which makes a significant contribution to the model. Even without including NIC, extremely low non-zero doses arise from distant rural residents. However, including NIC gives more cases and greater statistical power.

For all solid cancers and most individual sites, timesince-exposure was a better predictor than age-atexposure. The fitted dependence of risk on since is not governed by a latency threshold, and for all solid cancers, RR decreases with since at low doses. Risk could rise with since $<12.4$ years, the minimum value in the cohort.

Shuryak [32] attributes childhood radiation risk to initiation when cells are proliferating, while older adult risk arises from progression of previously acquired mutations. For all solid cancers, RR increases with agex at low doses, suggesting that for women, low dose radiation is acting primarily via progression.

The risks found here are surprising, as linear extrapolation from [1] predicts much lower values at $10 \mathrm{mGy}$. Preston's Table 10 shows female ERR $=0.58 /$ Gy with 
$90 \%$ CI $(0.43,0.69)$ at age 70 and agex 30 for solid cancers in the $0-4$ Gy $(+\mathrm{NIC})$ dose range (denoted here as $\mathrm{J}^{+}$). Adjusting for age-at-exposure, at agex 35 $\left.E R R\right|_{1 \text { Gy }}=0.53(0.37,0.67)$. Linear extrapolation gives $\left.E R R\right|_{10 \mathrm{mGy}}=0.0053(0.0037,0.0067)$. Over $\mathrm{B}^{+}$, the ecdos model P5se is preferred by method 1 ; at age 70 and since $\left.35 \mathrm{RR}\right|_{10 \mathrm{mGy}}=1.068(1.018,1.120)$, with ERR $\left.\right|_{10 \mathrm{mGy}}$ 12.8 times the extrapolated value. As Table 4 shows, in $\mathrm{A}^{+-}, \mathrm{B}^{+-}$, and $\mathrm{C}^{+-}$, the four selection methods choose ecdos models in 22 out of 24 cases, $90 \%$ CIs for ERR $\left.\right|_{10 \text { mGy }}$ exclude 0 in those cases and are borderline in 2 cases, and in 23 cases the resulting estimates of
ERR $\left.\right|_{10 \text { mGy }}$ are 9.6 - 45.7 times the prediction from linear extrapolation.

However, the GAM Poisson models give estimates comparable with [1] when fitted over $\mathrm{J}^{+}$and evaluated at $1 \mathrm{~Gy}$. Over this range, the preferred model (method 1, 2 or 3 ) is P5sd. At 1 Gy, age 70 and since $35, R R=1.80(1.60,2.03)$ while for P5ad at agex $35 \mathrm{RR}=1.56(1.40,1.73)$, close to Preston's estimate and CI. The dose response in $\mathrm{J}^{+}$for P5ae, P5ad, P5se, or P5sd is roughly comparable with the gender-averaged ERR shown in [1] (Fig. 3, using smoothed category estimates from the $0-2$ Gy data). Similar results are obtained over $\mathrm{J}^{-}$(excluding NIC).

Table 4 Estimates of ERR at 10 mGy, age 70, agex 35, since 35

\begin{tabular}{|c|c|c|c|c|c|c|c|}
\hline Sex & Range & Method & Model & Scale & Covariates & $\begin{array}{l}\text { ERR } 10 \text { mGy, Female } \\
90 \% \text { Cl, age } 70, \\
\text { agex } 35 \text {, since } 35\end{array}$ & Ratio \\
\hline MF & 0-4 Gy & & LinExt from [1] & 1 & $\mathrm{~s}, \mathrm{c}, \mathrm{d}, \mathrm{dose}$,age,agex & $0.0053(0.0037,0.0067)$ & 1.0 \\
\hline MF & 0-0.5 Gy (urban) & & WtMovAv from [2] & 1 & s,dose,age,agex & $0.02^{\mathrm{a}}$ (estimate) & 3.8 \\
\hline $\mathrm{F}$ & $A^{-}$ & 1 & P5se & 1 & $c, d, e c d o s, a g e$,since & $0.242(0.057,0.459)$ & 45.7 \\
\hline $\mathrm{F}$ & $A^{-}$ & 2 & P4se & 1 & c,d,ecdos,since & $0.225(0.045,0.437)$ & 42.5 \\
\hline $\mathrm{F}$ & $A^{-}$ & 3 & P4se & 1 & $c, d, e c d o s$, since & $0.225(0.045,0.437)$ & 42.5 \\
\hline $\mathrm{F}$ & $A^{-}$ & 4 & Q4se & 0.901 & $c, d, e c d o s$, since & $0.228(0.055,0.430)$ & 43.0 \\
\hline $\mathrm{F}$ & $\mathrm{A}^{+}$ & 1 & P5se & 1 & $c, d, e c d o s, a g e$, since & $0.230(0.054,0.436)$ & 43.4 \\
\hline $\mathrm{F}$ & $\mathrm{A}^{+}$ & 2 & P5se & 1 & $c, d, e c d o s, a g e$, since & $0.230(0.054,0.436)$ & 43.4 \\
\hline $\mathrm{F}$ & $\mathrm{A}^{+}$ & 3 & P4se & 1 & $c, d, e c d o s$, since & $0.202(0.032,0.401)$ & 38.1 \\
\hline $\mathrm{F}$ & $\mathrm{A}^{+}$ & 4 & Q4sd & 0.868 & $c, d, d o s e$, since & $0.088(-0.001,0.185)$ & 16.6 \\
\hline $\mathrm{F}$ & $\mathrm{B}^{-}$ & 1 & P5se & 1 & $c, d, e c d o s$,age,since & $0.088(0.017,0.164)$ & 16.6 \\
\hline $\mathrm{F}$ & $B^{-}$ & 2 & P4se & 1 & c,d,ecdos,since & $0.085(0.015,0.160)$ & 16.0 \\
\hline $\mathrm{F}$ & $B^{-}$ & 3 & P4se & 1 & $c$, d,ecdos,since & $0.085(0.015,0.160)$ & 16.0 \\
\hline $\mathrm{F}$ & $B^{-}$ & 4 & Q4se & 1.141 & c,d,ecdos,since & $0.077(0.011,0.148)$ & 14.5 \\
\hline $\mathrm{F}$ & $\mathrm{B}^{+}$ & 1 & P5se & 1 & $c, d, e c d o s$,age, since & $0.068(0.021,0.117)$ & 12.8 \\
\hline $\mathrm{F}$ & $\mathrm{B}^{+}$ & 2 & P3d & 1 & $c, d$,dose,age & $0.013(0.000,0.026)$ & 2.5 \\
\hline $\mathrm{F}$ & $\mathrm{B}^{+}$ & 3 & P2e & 1 & c,d,ecdos & $0.061(0.015,0.110)$ & 11.5 \\
\hline $\mathrm{F}$ & $\mathrm{B}^{+}$ & 4 & Q2e & 1.102 & c,d,ecdos & $0.061(0.013,0.112)$ & 11.5 \\
\hline $\mathrm{F}$ & $\mathrm{C}^{-}$ & 1 & P5se & 1 & $c, d, e c d o s, a g e$,since & $0.064(0.038,0.090)$ & 12.1 \\
\hline $\mathrm{F}$ & $\mathrm{C}^{-}$ & 2 & P5ae & 1 & c,d,ecdos,age,agex & $0.068(0.045,0.091)$ & 12.8 \\
\hline $\mathrm{F}$ & $\mathrm{C}^{-}$ & 3 & P3e & 1 & c,d,ecdos,age & $0.055(0.034,0.076)$ & 10.4 \\
\hline $\mathrm{F}$ & $\mathrm{C}^{-}$ & 4 & Q3e & 1.079 & c,d,ecdos,age & $0.051(0.034,0.068)$ & 9.6 \\
\hline $\mathrm{F}$ & $\mathrm{C}^{+}$ & 1 & P5se & 1 & c,d,ecdos,age,since & $0.091(0.055,0.128)$ & 17.2 \\
\hline $\mathrm{F}$ & $\mathrm{C}^{+}$ & 2 & P4se & 1 & $c, d, e c d o s$, since & $0.057(0.040,0.075)$ & 10.8 \\
\hline $\mathrm{F}$ & $\mathrm{C}^{+}$ & 3 & P4se & 1 & $c, d, e c d o s$, since & $0.057(0.040,0.075)$ & 10.8 \\
\hline $\mathrm{F}$ & $\mathrm{C}^{+}$ & 4 & Q4se & 1.066 & $c, d, e c d o s$, since & $0.056(0.039,0.073)$ & 10.6 \\
\hline
\end{tabular}

Method Model Selection Method

Model for P5se etc. see Table 2

LinExt Linear Extrapolation

WtMovAv Weighted Moving Averages

Covariates s=sex, c=city, d=distcat

Ratio ERR estimate from model / ERR estimate by Linear Extrapolation from [1]

aestimate from [2] Fig. 1 
At $1 \mathrm{~Gy}$, the interaction of ecdos or dose with logage and agex from fitting P5a over $\mathrm{J}^{+}$is consistent with [1], whose Fig. 4 is comparable with Fig. 11 here. If agex or since is fixed, RR $\left.\right|_{1}$ Gy decreases with age. Preston's Fig. 6 (using smoothed category estimates) is comparable with the curve for 1000 mGy in Fig. 12 panel b (P5ad at age 70). At age $70,\left.R R\right|_{1 \text { Gy }}$ initially decreases with agex but rises when agex $>40$. Using P5sd, with better fit, at age $\left.70 \mathrm{RR}\right|_{1}$ Gy peaks at since 35 . Similar curves arise at age 50 , but with higher values of RR.

However, the interactions at lower doses (see Figs. 5, 6, and 8) conflict with the main model in [1], which has $R R$ decreasing with agex. At low doses, RR generally decreases with age when agex is fixed, but increases with agex when age is fixed. Likewise, above $100 \mathrm{mGy}$ in $\mathrm{C}^{+} \mathrm{RR}$ decreases with age when since is fixed, but increases with age in $\mathrm{A}^{+}$and $\mathrm{B}^{+}$. At age $70, \mathrm{RR}$ decreases with since in $\mathrm{A}^{+}$and $\mathrm{B}^{+}$and when since $>35$ in $\mathrm{C}^{+}$. In all the low dose ranges considered here, RR increases with agex. Even in $\mathrm{J}^{+}$, the curve for $100 \mathrm{mGy}$ does not decrease with agex (see Fig. 12 panel $b$ ) Thus, the decrease of RR with agex (when agex $<40)$ in [1] is specific to the higher dose range and the response at $500 \mathrm{mGy}$ or above.

Pierce and Preston [2] applied a simplified linear model to the $0-500 \mathrm{mSv}$ urban incidence data for both sexes, finding a higher response than linear extrapolation would predict. Their Fig. 1 shows a smoothed curve obtained as a weighted moving average of category estimates. The sexaveraged RR at agex 30 and $100 \mathrm{mSv} \sim 1.09$, from which $\left.\mathrm{RR}\right|_{\text {female, } 100 \mathrm{mSv}} \sim 1.12$ as the fitted model has ERR $\left.\right|_{\text {female }}$

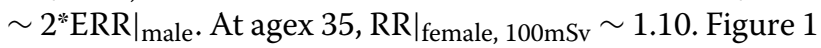
suggests that $\left.\mathrm{RR}\right|_{\text {female, }} 10 \mathrm{mSv} \sim 1.02$. By comparison, the preferred model (method 1 ) for the urban subcohort of $\mathrm{C}^{-}$ is P5se, and at age 70, since $35,\left.\mathrm{RR}\right|_{100 \mathrm{mGy}}=1.14$ (1.09, 1.18), while $\left.R R\right|_{10} \mathrm{mGy}=1.03(1.02,1.04)$. The interaction with since is very weak, and the preferred model with method 2 or 3 is P3e, for which at age $70 \mathrm{RR} \mid 100 \mathrm{mGy}=1.14$ $(1.09,1.19)$, as with the preferred quasi model Q3e. Models with either dose or ecdos show RR rising rapidly below
$150 \mathrm{mGy}$ before tapering, evidence of non-linearity in the urban data below $500 \mathrm{mGy}$.

Furukawa and co-workers [8] analysed the combined male and female incidence data below $400 \mathrm{mGy}$ with models including Linear Non Threshold, cubic smoothing spline, and a Bayesian semiparametric piecewise linear model, with dose responses at age 70 , agex 30 shown in their Fig. 1. The Bayesian and spline models gave similar responses at low doses. Whilst comparisons with the Bayesian model are beyond the scope of this paper, the spline model is roughly similar to P5ad, if dose knots are set at $0,5,20,40,60,80,100,125,150,175,200$, 250 , and $300 \mathrm{mGy}$. Furukawa analysed the individual data, but results using P5ad (with these knots) applied to the public data below $400 \mathrm{mGy}$ are not significantly different. Figure 13 (panel a) shows the gender averaged dose response for P5ad (knots). The spline estimate in [8] for $\left.E R R\right|_{100 \mathrm{mGy}}=0.03$ (CIs are not shown for this model) whilst for P5ad (knots) ERR $\left.\right|_{100 \mathrm{mGy}}=0.07(0.02,0.11)_{95 \%}$. The difference might result from the interaction structures in [8] which follow those in [1]. However, as Fig. 13 shows, the male response (panel b) lacks $95 \%$ significance below $\sim 300 \mathrm{mGy}$, but (panel c) female ERR $\left.\right|_{100 \mathrm{mGy}}=0.11$ $(0.04,0.19)$. Panel d shows the ecdos model P5ae with knots corresponding to those chosen for P5ad (knots). Now ERR $\left.\right|_{100} \mathrm{mGy}=0.17(0.10,0.24)$. With the female data, P5ae (knots) is preferred to P5ad (knots) by ML score and AIC. At 10 mGy with 95\% CIs, P5ad (knots) gives $\left.E R R\right|_{10 ~ m G y}=0.011(-0.001,0.023)$ whilst for P5ae (knots) $\left.\mathrm{ERR}\right|_{10 \mathrm{mGy}}=0.052(0.031,0.074)$.

The values listed in Table 4 are obtained from preferred models. P5ad is never selected, and P5ae is chosen only once. Table 4 largely reflects the predominant selection of ecdos models. Nevertheless, if P5ad and P5ae (with their default knot locations) are fitted over $\mathrm{A}^{+}$and evaluated at $10 \mathrm{mGy}$, age 70 and agex 35 , for $\mathrm{P} 5 \mathrm{ad} \mathrm{RR}=1.10$ with $90 \%$ CI $(1.00,1.21)$ while with P5ae RR $=1.21(1.04,1.41)$. P5ae is again preferred to P5ad by ML score and AIC. The default knots for P5ad in $\mathrm{A}^{+}$are $0,0.12,0.33,2.30,2.57$,
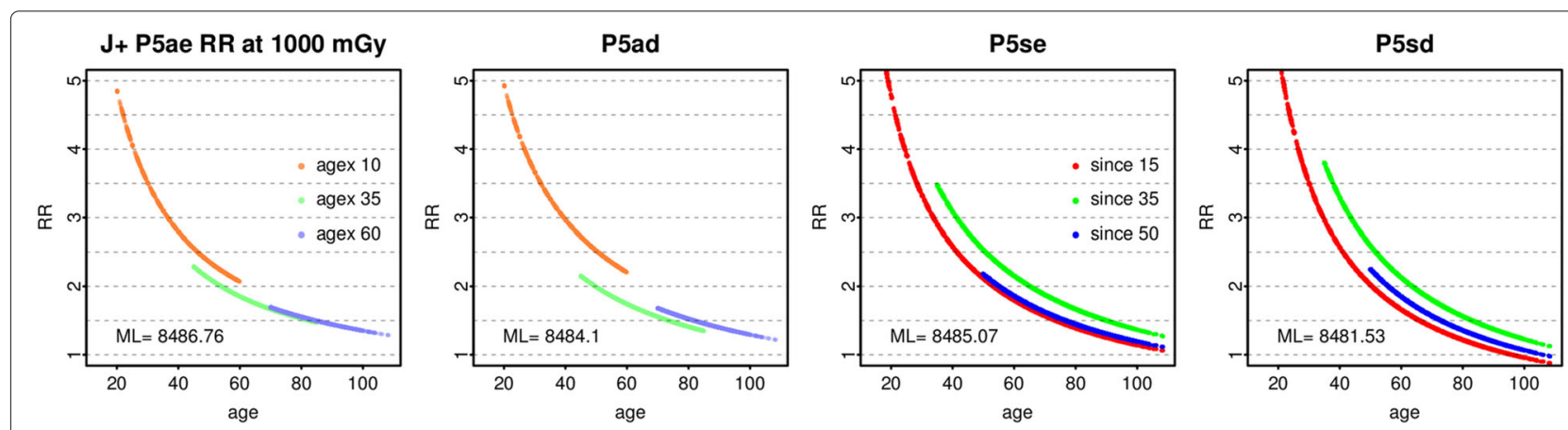

Fig. $110-4$ Gy interactions with age at 1 Gy. For all solid cancers in the full female dataset including NIC, as in [1], RR at 1000 mGy is plotted against age for the Poisson models P5ae and P5ad (Table 2), with agex fixed at 10, 35 and 60. Likewise RR at 1000 mGy is plotted against age for the Poisson models P5se and P5sd, with since fixed at 15, 35, and 50 

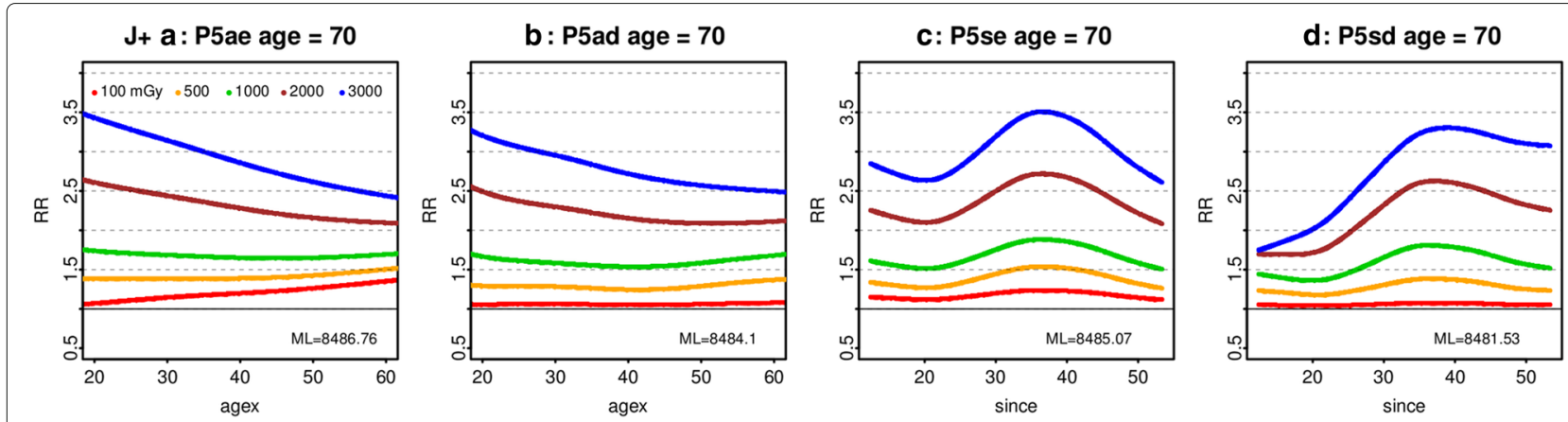

Fig. 120 - 4 Gy interactions with agex and since. For all solid cancers in the full female dataset including NIC, as in [1], RR at 100, 500, 1000, 2000 and 3000 mGy is plotted against agex (panels a and b) for the Poisson models P5ae and P5ad (Table 2), with age fixed at 70, and likewise against since (panels $\mathbf{c}$ and $\mathbf{d}$ ) for the Poisson models P5se and P5sd

$9.69,10.55,15.58$, giving a much richer structure than 0 , 5, 20. The estimate at $10 \mathrm{mGy}$ when fitting P5ad over $\mathrm{A}^{+}$is much higher than the estimate when fitting P5ad over $\mathrm{C}^{+}$.

Preston and co-workers [33] analysed the 1958-1998 solid cancer incidence data for exposure in utero or early childhood $(0-5$ years $)$, including NIC. For women exposed in early childhood, at attained age 50 $\left.E R R\right|_{1 \text { Sv }}=2.2(1.3,3.4)_{95 \%}$ (see [33] Table 5). Linear extrapolation is comparable with results from P5sd over $\mathrm{C}_{\mathrm{i}}{ }^{+}$(agex $\leq 5$, dose $\leq 500 \mathrm{mGy}$ ) at age 50 and since 45 , for which RR $\left.\right|_{10 \text { mGy }}=1.03(1.01,1.05) 95 \%$. With the preferred Poisson model (method 1) P5se, RR $\left.\right|_{10 \text { mGy }}=1.20$ $(1.06,1.35)_{95 \%}$. Methods 2 or 3 select P2e, giving RR $\left.\right|_{10}$ mGy $=1.14(1.05,1.24)_{95 \%}$, while method 4 chooses $\mathrm{Q} 2 \mathrm{e}$ giving $\left.\mathrm{RR}\right|_{10 \mathrm{mGy}}=1.14(1.06,1.22)_{95 \%}$.

For site-specific risks, some studies of other cohorts have found RR significantly raised after low dose exposure to low LET radiation. Some evidence from the literature on the thyroid, uterus and uterine corpus, is compared with results from quasipoisson models applied to appropriate subcohorts here.
Direct evidence on thyroid cancer risk arises from children given medical irradiation, residents affected by Chernobyl, nuclear workers, radiotherapy patients, and the A-bomb survivors. Except as indicated, these studies applied a linear ERR model.

Additional file 1: Table S9 shows some results from $[1,6,7,11,13,15,34-37]$. In each case, an appropriate subcohort of the female incidence data was analysed for comparison, using the simplest quasipoisson dose model Q2d and the preferred quasi model for the subcohort, chosen to approximate the dose and agex range in the study, or with mean dose close to that at which risk was reported.

In most cases, results from the subcohorts using either model are compatible with the reported estimates, as CIs overlap. There is striking agreement with the tinea capitis studies. Sadetzki [34] used a linear model which, at the mean dose, gives $\left.\mathrm{RR}\right|_{93 \mathrm{mGy} \text {, female }}=2.96$ (2.07, $4.31)_{95 \%}$. Fitting the quasi models over $\mathrm{C}^{+}$restricted to agex $<16$, Q2d gives RR $\left.\right|_{93 \text { mGy }}=2.30(1.36,3.89)_{95 \%}$, while the preferred model Q2e gives $\left.\mathrm{RR}\right|_{93 \mathrm{mGy}}=2.95$ $(2.05,4.24)_{95 \%}$. The earlier study by Ron [35] included
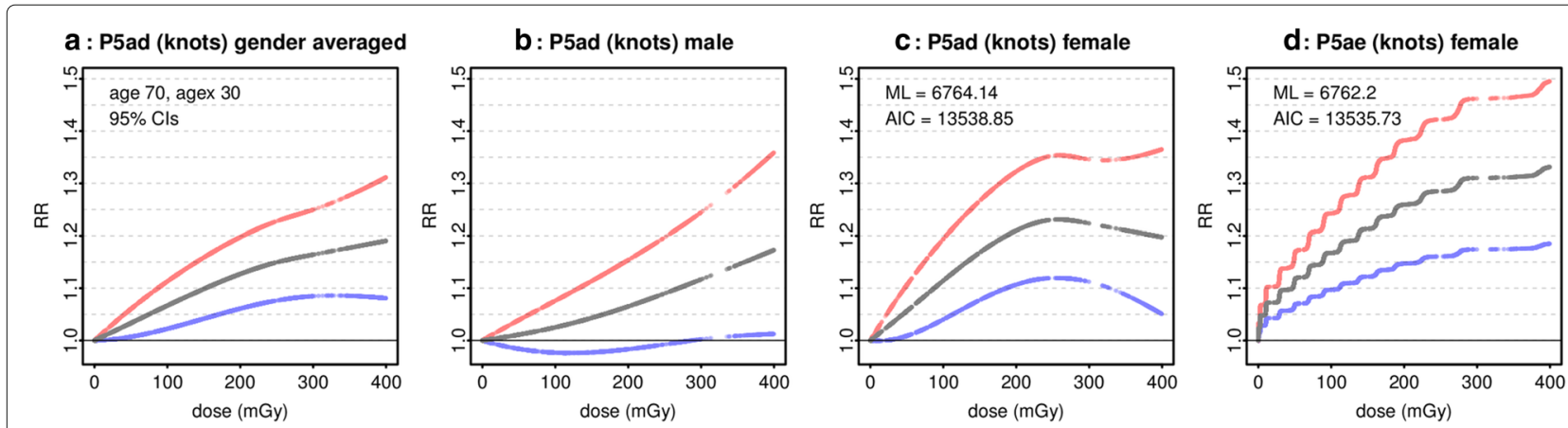

Fig. 130 - 400 mGy male and female. For comparison with [8], panel a shows the dose model P5ad (Table 2) fitted to the 0 - 400 mGy male-female data, with knots set at 0, 5, 20, 40, 60, 80, 100, 125, 150, 175, 200, 250, and $300 \mathrm{mGy}$. Gender averaged RR and 95\% Cls are shown at age 70 and agex 30. Panels $\mathbf{b}$ and $\mathbf{c}$ show the male and female responses. Panel $\mathbf{d}$ shows the ecdos model P5ae with corresponding knots at ecdf(dose)(0), ecdf(dose)(5) etc. ML scores and AIC for P5ad and P5ae fitted to the female data are shown for comparison 
the estimate $\left.R R\right|_{10 \mathrm{mGy}}=1.27(1.15,1.42)_{95 \%}$. Fitting the quasi models over $\mathrm{B}^{+}$restricted to agex $<16$, for which the mean py-weighted dose $=10.7 \mathrm{mGy}$, Q2d gives $\left.R R\right|_{10 \text { mGy }}=1.18(1.06,1.31)_{95 \%}$, and at the mean values of agex and age in [35] the preferred model Q5ad gives RR $\left.\right|_{10}$ mGy, agex 7.4, age $37.6=1.28(1.07,1.53)_{95 \%}$. These close agreements contrast with the differences seen in the pooled study [11], where the tinea capitis estimates appeared exceptionally high. However, the pooled estimates are compatible with GAM modelling on the appropriate subcohorts.

For Chernobyl survivors with agex $<18$, Little [6] considered a linear-exponential model with low-dose asymptote $\mathrm{ERR}=\alpha \mathrm{D}$, estimating $\alpha=9.72(2.67,94.31)_{95 \%}$. At the geometric mean dose as reported in [38], this gives an asymptotic $\left.R R\right|_{190 \mathrm{mGy}}=2.85(1.51,18.92)_{95 \%}$. Fitting the quasi models over $\mathrm{C}^{+}$restricted to agex $<18$, Q2d gives RR $\left.\right|_{190 \mathrm{mGy}}=2.62(1.50,4.57)_{95 \%}$. At the mean attained age from [39], the preferred model Q3e gives

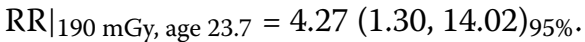

Sasaki [7] reanalysed the incidence data in [1], with a very different non-linear model. For the thyroid, from his Fig. 3(i) ERR $\left.\right|_{0.1 \mathrm{mSv} \text {, female }} \sim 0.35$, though no CI or interactions are shown. Results from $\mathrm{C}^{+}$appear compatible. The occupational study by Sont [13] did not estimate RR for female thyroid cancer, but found a Standardized Incidence Ratio $=1.42(1.19,1.69)_{90 \%}$. This was comparable with internal estimates of SIR (see "Methods"), with CIs by non-parametric bootstrap.

The results from GAM models applied to relevant subcohorts are compatible with $[6,11,13,15,34,35]$. They are higher than extrapolations from [1, 36, 37], which concerned higher dose ranges. However they appear compatible with [7].

Several studies found RR decreasing at higher doses. In the radiotherapy studies, doses were high and the downturn was only apparent above 6 Gy [5] or 10 Gy [36], whilst for Chernobyl survivors risk tapered above $1 \mathrm{~Gy}$ [6]. Using the no-interaction dose model Q2d, RR tapers above $400 \mathrm{mGy}$.

Unlike the thyroid, the uterus is not generally recognised as radiosensitive, although [1] notes an excess of cancers of the uterine corpus at young ages. Occupational studies [40-42] found significant excess mortality from uterine cancers in nuclear workers with a radiation record. The mortality rate increased with dose, but with few cases, mainly endometrial (corpus), ERR/Sv was not reported.

Analysing the National Registry for Radiation Workers, Muirhead [43] found that for mortality (internally calculated SMR) amongst solid cancers, only all uterine cancers showed trend with dose significant at the $5 \%$ level using a two-sided test. For incidence, there was a significant increasing trend with dose for endometrial cancer. RR was estimated with a linear model. For incidence of uterine ca
$\mathrm{ERR} / \mathrm{Sv}=10.523(0.27,39.4)_{90 \%}$. Near the mean dose $(24.9$ $\mathrm{mSv})$, this corresponds to $\left.\mathrm{RR}\right|_{25 \mathrm{mSv}}=1.26(1.01,1.99)$. For incidence of corpus ca, the Health Protection Agency [44] reported that Muirhead found ERR/Sv $=14.2(1.06$, 58.56) ${ }_{90 \%}$, corresponding to $\left.\mathrm{RR}\right|_{25 \mathrm{mSv}}=1.36(1.03,2.46)$.

For comparison, models were fitted over $\mathrm{B}^{+}$, restricted to $16 \leq$ agex $<60$. For the uterus, Q2d gives $\left.R R\right|_{25 \text { mGy }}=1.07(0.99,1.15)$. The preferred model Q3d has $\left.R R\right|_{25 \text { mGy, } 60 y r s}=0.96(0.75,1.23)$, while at age 65 $R R=1.23(0.98,1.54)$, and at age $70,1.29(1.04,1.60)$. For the corpus, Q2d gives $\left.R R\right|_{25}$ mGy $=1.08(0.89,1.31)$. The preferred model Q4se gives RR $\left.\right|_{25} \mathrm{mGy}$, since $30=5.24$ (1.22, $22.55)$, and $4.90(1.13,21.24)$ and $4.00(0.93,17.14)$ at since 25 and 35 respectively.

Davis [16] studied solid cancer incidence in the Techa River cohort, and found significant effects only for the oesophagus and uterus, using a linear model without interactions. Women constitute $57 \%$ of the cohort and age of entry begins at 0 . The mean dose was $53 \mathrm{mGy}$ and $90 \%$ of doses were below $125 \mathrm{mGy}$. For the uterus, mean age at diagnosis was 57 , and the result for ERR/100mGy = $0.21(0.01,0.51)_{95 \%}$ corresponds to $\left.\mathrm{RR}\right|_{50 \mathrm{mGy}}=1.11$ $(1.01,1.26)_{95 \%}$.

For comparison, models were fitted over $\mathrm{B}^{+}$. Q2d gives $\left.R R\right|_{50 \mathrm{mGy}}=1.37(1.09,1.72)_{95 \%}$. The preferred model Q5se gives RR $\left.\right|_{50 \mathrm{mGy}}$, age 60 , since $15=1.96(1.12,3.42)_{95 \%}$, and at since $=20,25,30$, and 35, the corresponding $\mathrm{RR}$ and $95 \% \mathrm{CI}=1.82(1.10,3.02), 1.67(1.01,2.76), 1.64$ $(0.99,2.73)$, and $1.70(1.02,2.84)$.

Using a linear model without effect modification, Preston [1] estimated for the uterus ERR/Gy $=0.10(-0.09$, $0.33)_{90 \%}$, and for the corpus ERR/Gy $=0.29(-0.14,0.95)$. However, for women with agex $<20$, ERR/Gy for the uterus $=0.37(0.001,0.86)$ and for the corpus 1.00 $(0.14,2.40)$. Near the py-weighted mean dose (85.3 mGy), with agex $<20$ the model predicts for the uterus $\left.\mathrm{RR}\right|_{80 \mathrm{mGy}}=1.03(1.00,1.07)_{90 \%}$ and for the corpus 1.08 $(1.01,1.19)$.

Fitting over $\mathrm{C}^{+}$restricted to agex $<20$, for the uterus Q2d gives $\left.R R\right|_{80 \text { mGy }}=1.79(1.19,2.68)_{90 \%}$. With the preferred model Q5se, RR $\left.\right|_{80} \mathrm{mGy}$, age 50, since $30=2.64$ (1.17, 5.97) ${ }_{90 \%}$, and at since $=35,40,45$ and 50 the corresponding $\mathrm{RR}$ and $90 \% \mathrm{CI}=3.07(1.41,6.67), 4.51(2.05$, $9.94), 3.76(1.65,8.59)$, and $2.72(1.11,6.62)$. For the corpus Q2d gives $1.03(0.88,1.21)$. For the preferred model Q4ad, RR $\left.\right|_{80 \mathrm{mGy} \text {, agex } 10}=0.88(0.56,1.37)$, whilst at agex $12,14,16,18$ and 20 the corresponding values are 1.13 $(0.74,1.74), 1.59(1.05,2.43), 2.04(1.33,3.11), 2.24$ (1.40, $3.60)$, and $2.18(0.96,4.98)$, i.e. the response is higher after puberty.

I have not used another implementation of GAM or the fully Bayesian methods of MCMC. Confidence intervals, based on Wald-type CIs for the linear predictor, were validated by simulation only for Poisson modelling of all 
solid cancers. Although the models explain nearly $60 \%$ of the deviance for all solid cancers (in $\mathrm{B}^{+}$), the figures for individual cancer sites are lower, given the smaller case numbers. Minimum smoothing parameters imposed for some sites depended on a visual judgement of the output. The public data lacks information on other exposures which may interact with radiation. The effects could be due to confounding by some other factor linked to distance from the hypocentres, although modelling did control for urban, rural, or NIC location. The modelling was not designed to test a particular hypothesis for cellular mechanism, or for the higher risks for women or Nagasaki residents.

Although increased risk with age-at-exposure (at fixed attained age) was found for many sites, as Boice observed [45] the flash doses all occurred in August 1945, so age-atexposure is confounded with birth cohort and changes in diet, smoking habits or infection rates, may explain apparent age-at-exposure (or time-since-exposure) effects. Protracted or fractionated exposures may also have different effects.

The impact of radiation in this cohort may be affected by blast damage. Stewart and Kneale [46] investigated possible selection bias, as subjects were only eligible to enter the (mortality) cohort if they survived to 1950. Incidence data begins in 1958, with the minimum timesince-exposure exceeding 12 years.

Wing and Richardson [47] point out that the dose estimates, revised several times, also depend on "the ability to elicit accurate information on location, position and shielding [which] was affected not only by traumatization of the survivors and their domestic stigmatization but by their distrust of medical teams working under occupation forces [48]." The A-bombs annihilated Hiroshima and Nagasaki, destroying their social fabric.

For all these reasons this cohort may not predict risk elsewhere.

\section{Conclusion}

The Generalized Additive Model avoids potential problems in fitting a mis-specified parametric model. In fact, the linear model is an inadequate description of relative risk for cancer incidence in female Japanese Abomb survivors exposed to low dose radiation. Contrary to the interactions at high doses, for all solid cancers risk increases with age-at-exposure and decreases with time-since-exposure, suggesting that low dose radiation acts through progression of previously accumulated damage.

Time-since-exposure models were generally preferred to age-at-exposure models. Transforming dose to its empirical cumulative distribution improved the model fit for all solid cancers and many specific sites. Constructing splines in this way improves their capacity to detect responses at very low doses. Covariate interactions were modelled with tensor product splines. Marginal Likelihood was the preferred method of smoothing parameter optimisation, and confidence intervals obtained from the Bayesian posterior covariance performed well.

The results at $10 \mathrm{mGy}$ are much higher than expected. Whilst higher female cancer risks and the highly skewed distribution of dose are recognised, significantly raised Relative Risk for women exposed to low doses, not found for all solid cancers in men, is new. Most other A-bomb survivor studies consider much wider dose ranges.

Whilst the results may be specific to this cohort, some studies of other cohorts with other methods give comparable results, notably for thyroid cancer where estimates are consistent with those derived here, and for the uterus.

Direct epidemiological evidence of risks from doses around $10 \mathrm{mGy}$ is a current research priority [18]. Risk estimates derived here from low dose data are much higher than those on which the ICRP has relied when setting recommended annual dose limits [4], including the annual occupational dose limit of $20 \mathrm{mSv}$. For all solid cancers, almost all estimates here of Excess Relative Risk for women at $10 \mathrm{mGy}$ are $9.6-45.7$ times the prediction by linear extrapolation from [1].

Generalized additive models applied to the low dose data for female Japanese A-bomb survivors show significant Relative Risks, particularly if the heavily skewed doses are transformed to improve their distribution. Evidence of elevated risk at low doses should contribute to higher radiation risk estimates and improve radiation protection for women.

\section{Additional file}

Additional file 1: Additional files are linked from a mini-website available with the online version of this paper. They consist of 1 PDF file with the Appendices, 10 images, 1 Excel workbook with 9 tables, 4 code files, 2 data files, and 6 output files. (ZIP $3727 \mathrm{~kb}$ )

\footnotetext{
Abbreviations

$\chi^{2}$ : Pearson Chi-squared statistic; $\hat{\phi}$ : Pearson estimate of scale for quasipoisson model; $\mathrm{A}^{+-}: 0$ - $20 \mathrm{mGy}$ including (excluding) NIC subjects; $\mathrm{B}^{+-}: 0$ - $100 \mathrm{mGy}+-$ $\mathrm{NIC} ; \mathrm{C}^{+-}: 0$ - $500 \mathrm{mGy}+-\mathrm{NIC}$; CD: Cook's Distance; Cl: Bayesian posterior confidence interval; $D_{\delta}$ : data cells with dose $>\delta$; dfres: residual degrees of freedom; e.d.f.: effective degrees of freedom; ERR: Excess Relative Risk; ecdf: empirical cumulative distribution function; ecdos: empirical cumulative distribution of dose; GAM: Generalized Additive Model; GCV.Cp: Generalized Cross Validation/Mallows Cp; J+- 0 - 4 Gy +- NIC; LCL: Bayesian posterior lower confidence limit; ML: Marginal Likelihood; NIC: Not In City; REML: Restricted Maximum Likelihood; RERF: Radiation Effects Research Foundation; RR: Relative Risk; $S_{\delta}$ : dose $>\delta$ and since $<35 ; T_{\delta}$ : dose $>\delta$ and agex $>35 ; U C L$ : Bayesian posterior upper confidence limit
}

\section{Acknowledgments}

Thanks to Simon Wood for help with GAMs and mgcv, and to David Richardson for encouragement. Thanks to the reviewers Richard Clapp and Kyoji Furukawa for editorial comments, and for alerting me to Dr Furukawa's analysis. 
The data described in this report were obtained from the Radiation Effects Research Foundation (RERF) in Hiroshima, Japan. RERF is a private foundation that is funded equally by the Japanese Ministry of Health and Welfare and the US Department of Energy through the US National Academy of Sciences. The conclusions reached in this report are mine and do not necessarily reflect the scientific judgement of RERF or its funding agencies.

\section{Funding}

This work was not funded.

\section{Availability of data and materials}

The dataset supporting the conclusions of this article is available in the Radiation Effects Research Foundation Downloadable Data respository, as the Life Span Study Solid Cancer Incidence Data, 1958-1998 and can be downloaded from http://www.rerf.jp/library/dl_e/ lssinc07.html

Some extracts from the dataset are included within the article's additional files.

\section{Authors' contributions}

GD designed the study and carried out the computations.

\section{Competing interests}

The authors declare that they have no competing interests.

\section{Consent for publication}

Not applicable.

\section{Ethics approval and consent to participate}

The study involves only freely available anonymous public data and published literature. I have not sought further Ethics Approval.

\section{Received: 5 July 2016 Accepted: 26 October 2016}

Published online: 24 November 2016

\section{References}

1. Preston DL, Ron E, Tokuoka S, Funamoto S, Nishi N, Soda M, et al. Solid cancer incidence in atomic bomb survivors: 1958 - 1998. Radiat Res. 2007;168:1-64.

2. Pierce $D A$, Preston DL. Radiation-related cancer risks at low doses among atomic bomb survivors. Radiat Res. 2000;154:178-86.

3. Blue Book: EPA Radiogenic Cancer Risk Models and Projections for the U.S. Population. Washington DC: U.S. Environmental Protection Agency, Office of Radiation and Indoor Air. 2011. EPA 402-R-11-001.

4. ICRP. 2007 Recommendations of the International Commission on Radiological Protection (Users Edition): ICRP Publication 103 (Users Edition). Ann ICRP. 2007;37(2-4).

5. Ronckers CM, Sigurdson AJ, Stovall M, Smith SA, Mertens AC, Liu Y, et al. Thyroid cancer in childhood cancer survivors: a detailed evaluation of radiation dose response and its modifiers. Radiat Res. 2006;166:618-28.

6. Little MP, Kukush AG, Masiuk SV, Shklyar S, Carroll RJ, Lubin JH, et al. Impact of uncertainties in exposure assessment on estimates of thyroid cancer risk among Ukrainian children and adolescents exposed from the Chernobyl accident. PLoS One. 2014;e85723:9.

7. Sasaki MS, Tachibana A, Takeda S. Cancer risk at low doses of ionizing radiation: artificial neural networks inference from atomic bomb survivors. J Radiat Res. 2014;55:391-406.

8. Furukawa K, Misumi M, Cologne J, Cullings H. A bayesian semiparametric model for radiation dose-response estimation. Risk Anal. 2016;36:1211-23.

9. Stewart AS, Webb J, Giles D, Hewitt D. Malignant disease in childhood and diagnostic irradiation in utero. Lancet. 1956;268(6940):447.

10. Wing S, Shy CM, Wood JL, Wolf S, Cragle DL, Frome EL. Mortality among workers at Oak Ridge National Laboratory: evidence of radiation effects in follow-up through 1984. JAMA. 1991;265:1397-402.

11. Ron E, Lubin JH, Shore RE, Mabuchi K, Modan B, Pottern LM, et al. Thyroid cancer after exposure to external radiation: a pooled analysis of seven studies. Radiat Res. 1995;141:259-77.

12. Richardson DB, Wing S. Greater sensitivity to ionizing radiation at older age: follow-up of workers at Oak Ridge National Laboratory through 1990. Int J Epidemiol. 1999;28:428-36.

13. Sont WN, Zielinski JM, Ashmore JP, Jiang $H$, Krewski D, Fair ME, et al. First analysis of cancer incidence and occupational radiation exposure based on the National Dose Registry of Canada. Am J Epidemiol. 2001;153:309-18.

14. Cardis E, Vrijheid M, Blettner M, Gilbert E, Hakama M, Hill C, et al. Risk of cancer after low doses of ionising radiation: retrospective cohort study in 15 countries. BMJ. 2005;331(7508):77.

15. Kopecky KJ, Stepanenko V, Rivkind N, Voillequé P, Onstad L, Shakhtarin $V$, et al. Childhood thyroid cancer, radiation dose from Chernobyl, and dose uncertainties in Bryansk Oblast, Russia: a population-based case-control study. Radiat Res. 2006;166:367-74.

16. Davis FG, Krestinina LY, Preston D, Epifanova S, Degteva M, Akleyev A Solid cancer incidence in the Techa River incidence cohort: 1956-2007. Radiat Res. 2015;184:56-65.

17. Brenner DJ, Doll R, Goodhead DT, Hall EJ, Land CE, Little JB, et al. Cancer risks attributable to low doses of ionizing radiation: assessing what we really know. Proc Natl Acad Sci U S A. 2003;100:13761-6.

18. NAS Nuclear and Radiation Studies Board. Planning towards the BEIR VIII report. 2014. http://dels.nas.edu/Past-Events/Planning-Towards-BEIR-VIIIReport/AUTO-0-14-84-B?bname=nrsb. Accessed 20 Oct 2016.

19. Hastie T, Tibshirani R. Generalized additive models (with discussion). Statist Sci. 1986;1:297-318.

20. R Core Team. R: a language and environment for statistical computing. Vienna: R Foundation for Statistical Computing; 2013. http://www.Rproject.org. Accessed 25 Sept 2013.

21. Wood SN. Generalized Additive Models: an introduction with R. Boca Raton, London, New York: Chapman \&. Hall/CRC: Taylor \& Francis Group; 2006

22. Life Span Study Solid Cancer Incidence Data, 1958-1998. Hiroshima, Japan: RERF; 2007. http://www.rerf.jp/library/dl_e/lssinc07.html. Accessed 20 Feb 2010.

23. Radiation Effects Research Foundation (RERF). A Cooperative Japan-US Research Foundation. http://www.rerf.jp. Accessed 20 Oct 2016.

24. Wood SN. Fast stable restricted maximum likelihood and marginal likelihood estimation of semiparametric generalized linear models. J R Stat Soc Series B Stat Methodol. 2011;73:3-36.

25. Sasaki MS, Nomura T, Ejima Y, Utsumi H, Endo S, Saito I, et al. Experimental derivation of relative biological effectiveness of A-bomb neutrons in Hiroshima and Nagasaki and implications for risk assessment. Radiat Res. 2008;170:101-17.

26. DiCiccio TJ, Efron B. Bootstrap confidence intervals. Statist Sci. 1996;11: 189-228.

27. UNSCEAR. Report to the General Assembly, with scientific annexes. New York: United Nations Scientific Committee on the Effects of Atomic Radiation. 2012;2012.

28. Preston DL, Shimizu Y, Pierce DA, Suyama A, Mabuchi K. Studies of mortality of atomic bomb survivors. Report 13: solid cancer and noncancer disease mortality: 1950-1997. Radiat Res. 2003;160:381-407.

29. National Research Council. Committee to Assess Health Risks from Exposure to Low Levels of lonizing Radiation. Health risks from exposure to low levels of ionizing radiation: BEIR VII Phase 2. Washington DC: National Academies Press; 2006

30. Nakashima E. Radiation dose response estimation with on low dose range using restricted cubic splines: application to all solid cancer mortality data, 1950-2003, in atomic bomb survivors. Health Phys. 2015;109:15-24.

31. Beels L, Bacher K, De Wolf D, Werbrouck J, Thierens H. gamma-H2AX foci as a biomarker for patient $X$-ray exposure in pediatric cardiac catheterization: are we underestimating radiation risks? Circulation. 2009;120:1903-9.

32. Shuryak I, Sachs RK, Brenner DJ. Cancer risks after radiation exposure in middle age. J Natl Cancer Inst. 2010;102:1628-36.

33. Preston DL, Cullings H, Suyama A, Funamoto S, Nishi N, Soda M, et al. Solid cancer incidence in atomic bomb survivors exposed in utero or as young children. J Natl Cancer Inst. 2008;100:428-36.

34. Sadetzki S, Chetrit A, Lubina A, Stovall M, Novikov I. Risk of thyroid cancer after childhood exposure to ionizing radiation for tinea capitis. J Clin Endocrinol Metab. 2006;91:4798-804.

35. Ron E, Modan B, Preston D, Alfandary E, Stovall M, Boice JDJ. Thyroid neoplasia following low-dose radiation in childhood. Radiat Res. 1989;120:516-31.

36. Bhatti $P$, Veiga LHS, Ronckers CM, Sigurdson AJ, Stovall M, Smith SA, et al. Risk of second primary thyroid cancer after radiotherapy for a childhood cancer in a large cohort study: an update from the childhood cancer survivor study. Radiat Res. 2010;174:741-52. 
37. Richardson DB. Exposure to ionizing radiation in adulthood and thyroid cancer incidence. Epidemiology. 2009;20:181-7.

38. Likhtarov I, Kovgan L, Masiuk S, Talerko M, Chepurny M, Ivanova O, et al. Thyroid cancer study among Ukrainian children exposed to radiation after the Chornobyl accident: improved estimates of the thyroid doses to the cohort members. Health Phys. 2014;106:370-96.

39. Tronko MD, Howe GR, Bogdanova TI, Bouville AC, Epstein OV, Brill AB, et al. A cohort study of thyroid cancer and other thyroid diseases after the Chornobyl accident: thyroid cancer in Ukraine detected during first screening. J Natl Cancer Inst. 2006;98:897-903.

40. Fraser P, Carpenter L, Maconochie N, Higgins C, Booth M, Beral V. Cancer mortality and morbidity in employees of the United Kingdom Atomic Energy Authority, 1946-86. Br J Cancer. 1993;67:615-24.

41. Carpenter L, Higgins C, Douglas A, Fraser P, Beral V, Smith P. Combined analysis of mortality in three United Kingdom nuclear industry workforces, 1946-1988. Radiat Res. 1994;138:224-38.

42. Atkinson WD, Law DV, Bromley KJ, Inskip HM. Mortality of employees of the United Kingdom Atomic Energy Authority, 1946-97. Occup Environ Med. 2004;61:577-85.

43. Muirhead CR, O'Hagan JA, Haylock RG, Phillipson MA, Willcock T, Berridge $\mathrm{GL}$, et al. Mortality and cancer incidence following occupational radiation exposure: third analysis of the National Registry for Radiation Workers. Br J Cancer. 2009;100:206-12.

44. Independent Advisory Group on lonising Radiation. Risk of Solid Cancers following Radiation Exposure: Estimates for the UK Population. Health Protection Agency: Chilton; 2011.

45. Boice, JD Jr. Models, models everywhere-is there a fit for lifetime risks. J Natl Cancer Inst. 2010;102:1606-9.

46. Stewart AM, Kneale GW. A-bomb survivors: factors that may lead to a re-assessment of the radiation hazard. Int J Epidemiol. 2000;29:708-14.

47. https://www.ncbi.nlm.nih.gov/pmc/articles/PMC1241119/pdf/ehp0110a00739.pdf.

48. Lindee MS. Suffering made real: American science and the survivors of Hiroshima. Chicago: University of Chicago Press; 1994.

\section{Submit your next manuscript to BioMed Central and we will help you at every step:}

- We accept pre-submission inquiries

- Our selector tool helps you to find the most relevant journal

- We provide round the clock customer support

- Convenient online submission

- Thorough peer review

- Inclusion in PubMed and all major indexing services

- Maximum visibility for your research

Submit your manuscript at www.biomedcentral.com/submit 